Artículo científico

(Original paper)

\title{
LAS ARAÑAS (ARACHNIDA: ARANEAE) DE LOS MÉDANOS DE SAMALAYUCA, CHIHUAHUA Y NUEVOS REGISTROS DE ESPECIES PARA MÉXICO
}

\section{SPIDERS (ARACHNIDA: ARANEAE) OF THE MEDANOS OF SAMALAYUCA, CHIHUAHUA AND NEW RECORDS OF SPECIES FOR MEXICO}

\author{
MA. Luisa JIMÉNEZ ${ }^{*}$, CARlos PALACIOS-CARDIEL ${ }^{1}$, IRVING DAVID CHÁVEZ-LARA ${ }^{2}$ \\ ${ }^{1}$ Laboratorio de Aracnología y Entomología, Centro de Investigaciones Biológicas del Noroeste, Instituto Politécnico Nacional \\ 195, Col. Playa Palo de Santa Rita Sur, La Paz, Baja California Sur, México. C.P. 23096. <ljimenez04@cibnor.mx>; \\ <palacios04@cibnor.mx> \\ ${ }^{2}$ Laboratorio de Ecología y Diversidad Animal, Universidad Autónoma de Ciudad Juárez, Anillo Envolvente del PRONAF y \\ Estocolmo s/n, Ciudad Juárez, Chihuahua, México. C.P. 32310.<al111296@alumnos.uacj.mx> \\ *Autor corresponsal: <ljimenez04@ cibnor.mx>
}

Recibido: 14/05/2019; aceptado: 24/03/2020; publicado en línea: 10/06/2020

Editor responsable: Guillermo Ibarra Núñez

Jiménez, M. L., Palacios-Cardiel, C., Chávez-Lara, I. D. (2020) Las arañas (Arachnida: Araneae) de los Médanos de Samalayuca, Chihuahua y nuevos registros de especies para México. Acta Zoológica Mexicana (nueva serie), 36, 1-23.

https://doi.org/10.21829/azm.2020.3612205

RESUMEN. Se proporciona una lista con 77 especies de arañas del Área Natural Protegida (ANP) Médanos de Samalayuca, agrupadas en 51 géneros y 21 familias. Las familias con mayor número de especies fueron Salticidae, Thomisidae, Gnaphosidae y Philodromidae. También se aportan cinco nuevos registros de especies de arañas para la República Mexicana y ocho para el Estado de Chihuahua. El matorral desértico micrófilo agrupó a 16 familias 27 géneros 31 especies y de la vegetación del desierto arenoso se registraron 15 familias, 28 géneros y 37 especies, compartiendo 17 familias, 36 géneros y 46 especies, con una complementariedad del 84\%. En el estrato de vegetación se registraron 13 familias 43 especies y en el suelo 11 familias y 31 especies, compartiendo tres especies, con una complementariedad del $95 \%$.

Palabras clave: arañas de Chihuahua; arañas de Samalayuca; arañas de zonas áridas

Jiménez, M. L., Palacios-Cardiel, C., Chávez-Lara, I. D. (2020) Spiders (Arachnida: Araneae) of the Medanos of Samalayuca, Chihuahua and new records of species for Mexico. Acta Zoológica Mexicana (nueva serie), 36, 1-23.

https://doi.org/10.21829/azm.2020.3612205

\begin{abstract}
A faunistic spider list of the ANP Medanos de Samalayuca is provided with a total of 77 species, 51 genera and 21 families. The most diverse families are Salticidae, Thomisidae, Gnaphosidae and Philodromidae. We include information of thirteen new spider records; five are for Mexico and eight for Chihuahua State. Sixteen families, 27 genera and 31 species are reported for the macrophile desert shrub and 15 families, 28 genera and 37 species for the sandy desert vegetation. Complementarity between both
\end{abstract}


types of vegetation was $84 \%$. Thirteen families and 43 species and 11 families and 31 species were captured in vegetation and soil strata, respectively with a complementarity of $95 \%$. Both strata shared three species.

Key words: chihuahuan spiders; Samalayucan spiders; arid zones spiders

\section{INTRODUCCIÓN}

Las arañas de las regiones desérticas son uno de los grupos de animales más relevantes por su abundancia y gran adaptación a estos ambientes, además de que tienen un papel preponderante y fundamental en el flujo de energía en las cadenas alimentarias (Polis \& Hurd, 1995), por ser las principales depredadoras del grupo de insectos (Polis \& McCormick, 1986). Junto con otros artrópodos depredadores, forman una biomasa mayor que los vertebrados, que en conjunto son muy importantes en las cadenas tróficas en los desiertos (Polis \& Yamashita, 1991). La composición de las comunidades de arañas varía estructuralmente como respuesta a cualquier cambio o disturbio ambiental por lo que son consideradas indicadores de la calidad del ambiente (Wheater et al., 2000; Ibarra-Núñez, 2014).

El desierto Chihuahuense, el más extenso de Norteamérica, es considerado como el de mayor diversidad de América por su buen estado de conservación y alto grado de endemismo (CONANP, 2013). En este desierto se localiza el Área Natural Protegida (ANP) conocida como los Médanos de Samalayuca en el estado de Chihuahua, un lugar con un sistema complejo de dunas de arena sílica. Esta área es considerada una de las más importantes de nuestro país por tener un ecosistema relictual como resultado de los procesos erosivos milenarios durante el Triásico-Cretácico (CONANP, 2013). El ANP Médanos de Samalayuca se ubica en los municipios Juárez, Guadalupe Distrito y Bravo, así como una pequeña fracción del norte del municipio de Ahumada. En esta región la precipitación es mínima y el agua solo se encuentra en varios manantiales de agua subterránea que son vitales para el desarrollo de la actividad antropogénica (CONANP, 2013). El clima es similar al del norte del desierto chihuahuense, (BWKx'(e') templado y semidesértico (Schmidt, 1979; CONANP, 2013), con precipitación media anual de $212 \mathrm{~mm}$ (Álvarez, 1973) y temperatura media anual de $17.9^{\circ} \mathrm{C}$ con una máxima de $41.2^{\circ} \mathrm{C}$ y mínima de $-16^{\circ} \mathrm{C}(\mathrm{Schmidt} \&$ Marston, 1981).

La composición vegetal de la región está constituida por aproximadamente 248 especies de plantas en 36 familias, siendo las más diversas Poacea, Asteracea, Cactacea y Fabaceae (CONANP, 2013). En cuanto a la fauna, se registran 44 especies de reptiles (CONANP, 2013), 46 especies de aves (Ontiveros, 2011) y 62 especies de mamíferos (Anderson, 1972). De los artrópodos terrestres sólo se registran trabajos sobre ácaros y colémbolos (Cepeda \& Whitford, 1990), hormigas (MacKay et al., 1985) y sobre el consumo de 10 taxones de artrópodos en la dieta de una lagartija (Lemos-Espinal et al., 2003).

Del grupo de arañas no existe ningún inventario de esta región, solo dos descripciones de especies de arañas (Richman, 2008; Valdez-Mondragón et al., 2018) para los Médanos de Samalayuca. La mayoría de los registros son únicamente conocidos para el estado de Chihuahua, provienen de las recopilaciones bibliográficas de Hoffmann (1976) y Jiménez (1996) quienes indican la presencia de 100 y 168 especies respectivamente para esta entidad federativa. Esta pobreza de información demuestra que el conocimiento de las arañas es incipiente tanto para esta región como para el estado de Chihuahua. Por ello el objetivo de este trabajo es proporcionar un primer inventario faunístico de los Médanos de Samalayuca, así como dar a conocer los nuevos registros de arañas para México y para el estado de Chihuahua. 


\section{MATERIALES Y MÉTODOS}

Este trabajo formó parte del proyecto "Inventario multitaxonómico del ANP Médanos de Samalayuca" (PJ018 CONABIO), donde se seleccionaron cinco zonas de estudio, ubicadas entre los $31^{\circ} 23^{\prime} 3.93^{\prime \prime} \mathrm{N}$, $106^{\circ} 36^{\prime} 4.71^{\prime \prime} \mathrm{O}$ en el extremo Noreste y $31^{\circ} 6^{\prime} 24.90^{\prime \prime} \mathrm{N},-106^{\circ} 11^{\prime} 50.96^{\prime \prime} \mathrm{O}$ en el extremo Sureste de esta región (Fig. 1). La zona 1 representa una pequeña porción de la Sierra Presidio con vegetación dominante de matorral desértico micrófilo (MDM), una pequeña porción de matorral desértico rosetófilo (MDR) con representantes de Larrea tridentata (Sessé et Moc. ex DC.) y varias especies de Opuntia; la zona 2 se encuentra ubicada en la cara norte de la Sierra de Samalayuca y la zona 3 al sur de esta misma sierra, ambas con una composición vegetal de desierto arenoso (VDA), al sureste se presenta una pequeña porción de matorral desértico micrófilo y al norte de manera escasa, un pastizal inducido, es considerada en este trabajo como una zona de transición (MDM/VDA). Las zonas 4 y 5 están localizadas al centro y sureste de los Médanos de Samalayuca en un ecosistema relictual. La zona 4 se representa por una tercera parte de vegetación de desierto arenoso al oeste y al este sin vegetación aparente (SVA). La zona 5 comprende un 95\% de vegetación de desierto arenoso y al noreste sin vegetación aparente. Ambas zonas son consideradas como vegetación de desierto arenoso (VDA) (CONANP, 2013).
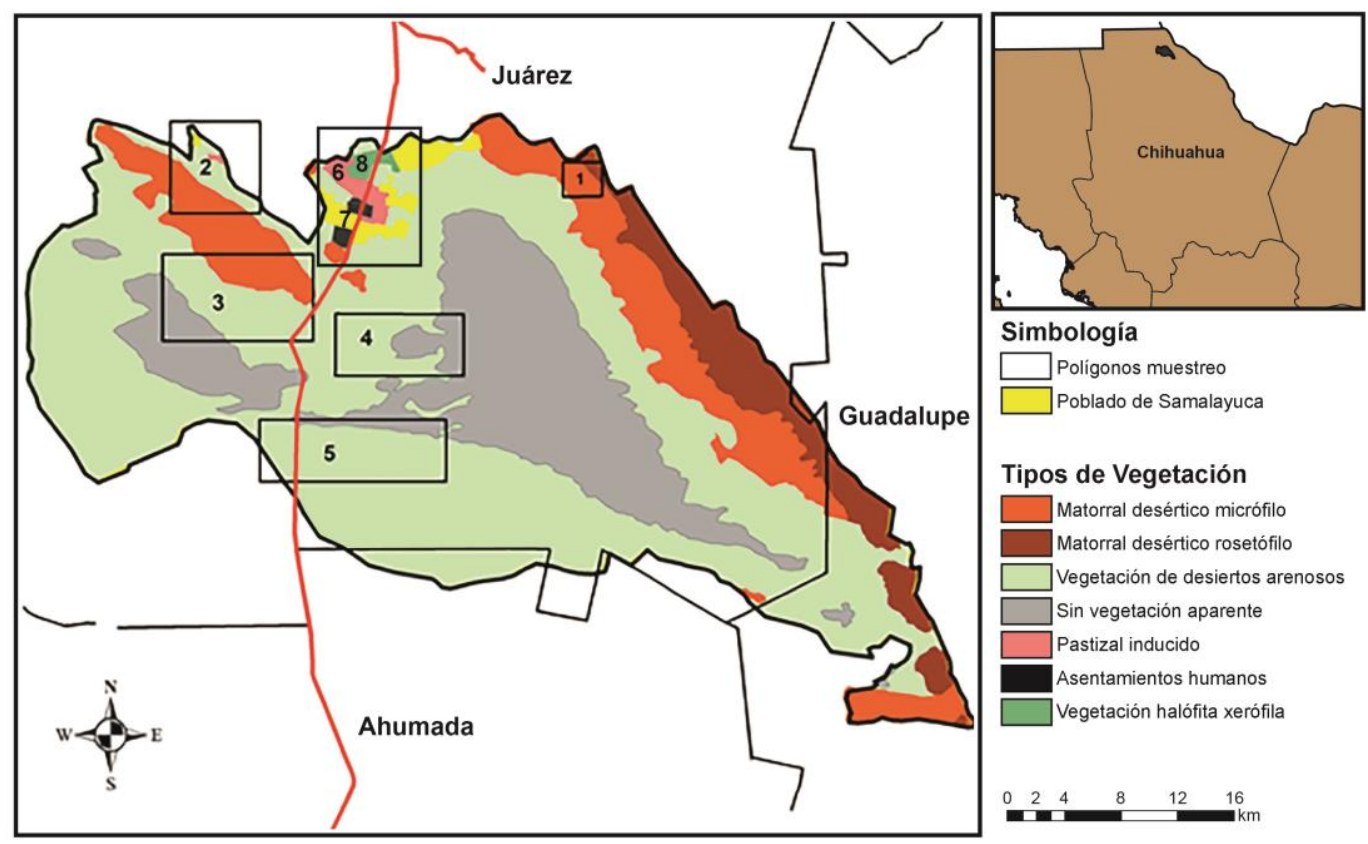

Figura 1. Sitios de recolecta de arañas en la ANP Médanos de Samalayuca, Chihuahua, México. Mapa modificado de CONANP 2013.

Las arañas fueron capturadas en tres expediciones durante el 2017. Del 15 al 30 de junio durante la época de secas, del 20 de julio al 4 de agosto en la época de lluvias y del 20 de septiembre al 4 de octubre en la época post-húmeda. En cada zona se trazó un transecto de $100 \mathrm{~m}$ al azar y perpendicular al camino principal por su fácil acceso y por seguridad personal, debido a los conflictos de inseguridad que hay en la región. A lo largo de cada transecto se colocaron 10 trampas de caída a intervalos de $10 \mathrm{~m}$ cada una, utilizando como conservador $250 \mathrm{ml}$ de etilénglicol al 50\%. Cada trampa consistió en un recipiente y un embudo de plástico de $10 \mathrm{~cm}$ de ancho por $20 \mathrm{~cm}$ de alto, en el interior se colocó un colector de plástico de $10 \mathrm{~cm}$ de alto por $7 \mathrm{~cm}$ de acho, donde se depositó el conservante. Las trampas fueron enterradas durante una semana a nivel del suelo y cubiertas con una malla metálica de $20 \times 20 \mathrm{~cm}$, separada de la orilla del embudo a $3 \mathrm{~cm}$, suspendida por 4 patas metálicas, una en cada esquina para apoyarse en el suelo. Después 
el contenido se retiró y se coló en otro recipiente con alcohol de caña al $70 \%$ para su transporte. Como complemento a las recolectas en el suelo, en el mismo transecto se hicieron capturas en el follaje durante el día de 8:00 a 12:00 h (4 horas), utilizando redes planas de manta de 1 x $1 \mathrm{~m}$, empleando una vara para golpear o sacudir la vegetación en un rango de 0.5-2 $\mathrm{m}$ de altura para hacer caer y capturar los organismos, durante aproximadamente 10-15 min para recolectarlos, utilizando aspiradores y pinceles y en un radio de $5 \mathrm{~m}$ en los mismos sitios donde fueron enterradas las trampas de caída. La ubicación de los transectos fueron los mismos en cada expedición. Conjuntamente también se hicieron recolectas manuales diurnas, durante una hora por zona, buscando en grietas, hendiduras, bajo rocas y con ayuda de pinzas. Estas recolectas fueron realizadas en cada zona por dos personas en la temporada de secas y por una persona en las temporadas húmeda y post-húmeda. Todos los ejemplares (juveniles y adultos) fueron separados e identificados a nivel de familia y género de acuerdo con Ubick et al. (2017) y a nivel específico utilizando trabajos o revisiones taxonómicas de diversos autores. Los juveniles fueron identificados a nivel genérico o específico cuando fue posible al compararlos con los adultos. Se ilustran con fotografías digitales únicamente los nuevos registros de géneros y especies para México y para el Estado de Chihuahua. Las fotografías fueron tomadas con cámaras Canon 5D Mark 2 DSLR (lente macro $100 \mathrm{~mm}$ L y flash Canon Twin Lite) y Sony Alpha NEX-5N. Previamente los genitales de las hembras y machos fueron aclarados con $\mathrm{KOH}$ durante cinco minutos. Los nombres de las especies fueron verificados con el catálogo mundial de arañas (World Spider Catalog, 2019) y los ejemplares fueron depositados en la Colección de Arácnidos (CARCIB B.C.-INV-193-01-07) del Centro de Investigaciones Biológicas del Noroeste (CIBNOR), Baja California Sur, México. Para obtener la estimación de la riqueza total de especies de arañas, se utilizó programa EstimateS versión 9.1 (Colwell, 2013) y dos estimadores de riqueza no paramétricos Chao 1 y ACE para construir la curva de acumulación de especies, para ver qué tan completo es el muestreo (Sørensen, 2003; Soberón \& Llorente, 1991). Para estimar la complementariedad de la composición entre los ambientes matorral desértico micrófilo (MDM) y la vegetación del desierto arenoso (VDA) y entre los estratos del suelo y vegetación, se utilizó el índice de Colwell y Coddington (1994) (CAB = UAB/SAB).

\section{RESULTADOS}

De la ANP Médanos de Samalayuca, se recolectó un total de 361 ejemplares pertenecientes a 21 familias, 51 géneros y 77 especies, de las cuales cinco especies son nuevos registros para México y ocho para el estado de Chihuahua (Cuadro 1).

Cuadro 1. Listado faunístico de las arañas presentes en Los Médanos de Samalayuca, Chihuahua, México. *Nuevo registro para México. **Nuevo registro para Chihuahua. Matorral desértico micrófilo (1), Ecotono (2, 3), Vegetación desierto arenoso $(4,5)$. Estrato $(E): S=$ suelo, $V=$ vegetación.

\begin{tabular}{|c|c|c|c|c|c|c|c|c|}
\hline Familia & Especie & 1 & 2 & 3 & 4 & 5 & Total & $\mathbf{E}$ \\
\hline Agelenidae & Agelenopsis aperta (Gertsch, 1934) & 5 & & & & & 5 & VS \\
\hline \multirow{4}{*}{ Araneidae } & Aculepeira packardi (Thorell, 1875) & & & 1 & & & 1 & $\mathrm{~V}$ \\
\hline & Araneus sp. & 1 & & & & & 1 & V \\
\hline & Argiope trifasciata (Forsskål, 1775) & & & & & 1 & 1 & V \\
\hline & Metepeira arizonica Chamberlin \& Ivie, $1942 * *$ & 3 & 5 & 3 & 3 & 2 & 16 & $\mathrm{~V}$ \\
\hline Corinnidae & Castianeira amoena (C.L. Koch, 1842)** & & & 1 & & & 1 & $\mathrm{~V}$ \\
\hline \multirow{4}{*}{ Dictynidae } & Dictyna sierra Chamberlin, 1948* & & 1 & & & & 1 & $\mathrm{~V}$ \\
\hline & Emblyna reticulata (Gertsch \& Ivie, 1936) & & & & 3 & & 3 & V \\
\hline & Emblyna sp. affin. melva (Chamberlin \& Gertsch, 1958) & & & & 3 & 1 & 4 & $\mathrm{~V}$ \\
\hline & Emblyna sp. & 4 & & & & & 4 & $\mathrm{~V}$ \\
\hline \multirow{3}{*}{ Diguetidae } & Diguetia canities $(\mathrm{McCook}, 1890)$ & & 1 & & & & 1 & $\mathrm{~V}$ \\
\hline & Diguetia imperiosa Gertsch \& Mulaik, 1940** & & 3 & 2 & & & 5 & V \\
\hline & Diguetia signata Gertsch, 1958 & 3 & 11 & 5 & 3 & & 22 & V \\
\hline
\end{tabular}




\begin{tabular}{|c|c|c|c|c|c|c|c|c|}
\hline Familia & Especie & 1 & 2 & 3 & 4 & 5 & Total & $\mathbf{E}$ \\
\hline \multirow[t]{2}{*}{ Filistatidae } & Filistatoides sp. & & 2 & 1 & 2 & & 5 & $\mathrm{~S}$ \\
\hline & Kukulcania arizonica (Chamberlin \& Ivie, 1935) & & & 1 & & & 1 & $\mathrm{~S}$ \\
\hline \multirow{8}{*}{ Gnaphosidae } & Callilepis титаi Platnick, 1975 & & & 1 & 1 & 1 & 3 & $\mathrm{~S}$ \\
\hline & Drassyllus mumai Gertsch \& Riechert, 1976** & 3 & & 5 & & & 8 & $\mathrm{~S}$ \\
\hline & Herpyllus ecclesiasticus Hentz, 1832** & 3 & & 3 & & & 6 & $\mathrm{~S}$ \\
\hline & Herpyllus sp. & 1 & & & & & 1 & $\mathrm{~S}$ \\
\hline & Micaria sp. & & & 1 & & & 1 & $\mathrm{~S}$ \\
\hline & Nodocion utus (Chamberlin, 1936) & & & 1 & & & 1 & $\mathrm{~S}$ \\
\hline & Zelotes lasalanus Chamberlin, 1928 & & & 1 & & & 1 & $\mathrm{~S}$ \\
\hline & Zelotes monachus Chamberlin, 1924 & & & 1 & & & 1 & $\mathrm{~S}$ \\
\hline Linyphiidae & Frontinella pyramitela (Walckenaer, 1841) & & & 1 & & & 1 & $\mathrm{~V}$ \\
\hline \multirow{6}{*}{ Lycosidae } & Camptocosa parallela (Banks, 1898) & 2 & & & & & 2 & VS \\
\hline & Hogna sp. & 2 & & & & & 2 & $\mathrm{~S}$ \\
\hline & Pardosa ramulosa (McCook, 1894) & & 3 & & & & 3 & $\mathrm{~S}$ \\
\hline & Pardosa sura Chamberlin \& Ivie, 1941 & 8 & & & & & 8 & $\mathrm{~S}$ \\
\hline & Pardosa grupo lapidicina & & & 1 & & & 1 & $\mathrm{~S}$ \\
\hline & Schizocosa mccooki (Montgomery, 1904) & 1 & & 3 & 3 & 5 & 12 & $\mathrm{~S}$ \\
\hline Mimetidae & Mimetus sp. & & & & & 1 & 1 & $\mathrm{~V}$ \\
\hline Miturgidae & Syspira sp. & 1 & 1 & & & & 2 & $\mathrm{~S}$ \\
\hline Oecobiidae & Oecobius putus O. Pickard-Cambridge, 1876 & & & 1 & & & 1 & $\mathrm{~S}$ \\
\hline \multirow{3}{*}{ Oxyopidae } & Hamataliwa sp. & & 2 & & & & 2 & $\mathrm{~V}$ \\
\hline & Oxyopes tridens Brady, 1964 & & & & & 1 & 1 & $\mathrm{~V}$ \\
\hline & Peucetia viridans (Hentz, 1832) & 2 & 4 & 1 & & & 7 & $\mathrm{~V}$ \\
\hline \multirow{7}{*}{ Philodromidae } & Apollophanes texanus Banks, 1904** & 1 & 1 & & & & 2 & $\mathrm{~V}$ \\
\hline & Philodromus arizonensis Dondale \& Redner, 1969* & & & 2 & & & 2 & $\mathrm{~V}$ \\
\hline & Philodromus infuscatus utus Chamberlin 1921 & & & & 2 & & 2 & V \\
\hline & Philodromus sp. & 1 & & & 1 & & 2 & $\mathrm{~V}$ \\
\hline & Rhysodromus histrio (Latreille, 1819) & & & 1 & 2 & 1 & 4 & $\mathrm{~V}$ \\
\hline & Titanebo mexicanus (Banks, 1898) & & & 2 & 1 & & 3 & $\mathrm{~V}$ \\
\hline & Titanebo dondalei (Sauer, 1968)* & & & 1 & 3 & & 4 & $\mathrm{~V}$ \\
\hline \multirow{4}{*}{ Pholcidae } & Modisimus sp. & 1 & & & & & 1 & $\mathrm{~S}$ \\
\hline & Physocyclus enaulus Crosby, 1926 & 2 & & & 2 & & 4 & $\mathrm{~S}$ \\
\hline & Psilochorus imitatus Gertsch \& Mulaik, 1940* & 2 & 5 & 10 & 2 & 5 & 24 & $\mathrm{~S}$ \\
\hline & Psilochorus sp. & 1 & & 9 & 6 & 2 & 18 & $\mathrm{~S}$ \\
\hline \multirow{13}{*}{ Salticidae } & Habronattus virgulatus Griswold, 1987 & & 1 & 1 & & 1 & 3 & $\mathrm{~V}$ \\
\hline & Habronattus conjunctus (Banks, 1898) & & 4 & 1 & 9 & 2 & 16 & $\mathrm{~V}$ \\
\hline & Habronattus klauseri (Peckham \& Peckham, 1901) & 1 & & & & & 1 & $\mathrm{~V}$ \\
\hline & Metacyrba taeniola similis Banks, 1904 & & & 2 & & & 2 & SV \\
\hline & Metaphidippus chera (Chamberlin, 1924) & & & 1 & 1 & & 2 & $\mathrm{~V}$ \\
\hline & Metaphidippus sp. & & & & 1 & & 1 & $\mathrm{~V}$ \\
\hline & Metaphidippus sp. grupo manni & & & & 1 & & 1 & V \\
\hline & Paramarpissa albopilosa (Banks, 1902) & & & & 8 & & 8 & $\mathrm{~V}$ \\
\hline & Phidippus apacheanus Chamberlin \& Gertsch, 1929 & & & & 1 & & 1 & $\mathrm{~V}$ \\
\hline & Sassacus papenhoei Peckham \& Peckham, 1895 & & & 1 & 1 & 1 & 3 & $\mathrm{~V}$ \\
\hline & Sassacus sp. affin. cyaneus (Hentz, 1846) & & & 1 & & & 1 & $\mathrm{~V}$ \\
\hline & Sassacus sp. affin. samalayucae Richman, 2008 & 3 & & 1 & & & 4 & V \\
\hline & Synageles noxiosus (Hentz, 1850) & & & 1 & & & 1 & $\mathrm{~V}$ \\
\hline Segestriidae & Ariadna sp. & 1 & & & & & 1 & $\mathrm{~S}$ \\
\hline Sicariidae & Loxoceles apachea Gertsch \& Ennik, 1983 & 7 & & & 1 & & 8 & $\mathrm{~S}$ \\
\hline Theraphosidae & Aphonopelma sp. & 2 & 1 & 1 & & 1 & 5 & $\mathrm{~S}$ \\
\hline \multirow{4}{*}{ Theridiidae } & Asagena medialis (Banks, 1898) & & & & 1 & 2 & 3 & $\mathrm{~S}$ \\
\hline & Asagena fulva (Keyserling, 1884) & & 1 & & & & 1 & $\mathrm{~S}$ \\
\hline & Euryopis sp. & & & & 1 & & 1 & $\mathrm{~S}$ \\
\hline & Latrodectus hesperus Chamberlin \& Ivie, 1935** & 2 & 5 & 4 & 9 & 6 & 26 & $\mathrm{~S}$ \\
\hline
\end{tabular}




\begin{tabular}{|c|c|c|c|c|c|c|c|c|}
\hline Familia & Especie & 1 & 2 & 3 & 4 & 5 & Total & $\mathbf{E}$ \\
\hline \multirow[t]{2}{*}{ Theridiidae } & Steatoda variata Gertsch, 1960 & 7 & & & & & 7 & $\mathrm{~S}$ \\
\hline & Steatoda sp. & & 2 & & 2 & & 4 & $\mathrm{~S}$ \\
\hline \multirow{10}{*}{ Thomisidae } & Mecaphesa californica (Banks, 1896) & & & & & 3 & 3 & $\mathrm{~V}$ \\
\hline & Mecaphesa celer (Hentz, 1847) & & & & 5 & & 5 & $\mathrm{~V}$ \\
\hline & Mecaphesa coloradensis (Gertsch, 1933)** & 5 & & 2 & 14 & & 21 & V \\
\hline & Mecaphesa dubia (Keyserling, 1880) & & 1 & & & & 1 & $\mathrm{~V}$ \\
\hline & Mecaphesa rothi (Schick, 1965)* & 6 & & 2 & 2 & 3 & 13 & V \\
\hline & Mecaphesa sp. & 9 & & & & & 9 & V \\
\hline & Xysticus gertschi Schick, 1965 & & & & 6 & & 6 & V \\
\hline & Xysticus lutzi Gertsch, 1935 & 1 & & & & & 1 & $\mathrm{~S}$ \\
\hline & Total & 91 & 54 & 77 & 100 & 39 & 361 & \\
\hline & Número de especies & 31 & & 46 & & 37 & & \\
\hline
\end{tabular}

Las familias con mayor de riqueza de especies fueron Salticidae con 13 especies, Thomisidae y Gnaphosidae con ocho especies, respectivamente, y Philodromidae con siete, contrastando con las familias Agelenidae, Corinnidae, Linyphiidae, Mimetidae, Miturgidae, Oecobiidae, Segestriidae, Sicaridae y Theraphosidae, representadas solamente por una especie (Fig. 2). A nivel genérico Salticidae fue la más diversa con siete géneros, seguida de Gnaphosidae con seis, Araneidae, Lycosidae, Philodromidae y Theridiidae con cuatro géneros, respectivamente (Fig. 2).

Los géneros más ricos en especies fueron Mecaphesa (Thomisidae) con seis, seguido por Emblyna (Dictynidae) y Pardosa (Lycosidae) con cuatro, y Philodromus (Philodromidae), Habronattus, Metaphidippus y Sassacus (Salticidae) con tres especies, respectivamente; el resto de los géneros tuvieron de una a dos especies (Cuadro 1).

Las familias más abundantes fueron: Thomisidae (16.3\%), Pholcidae (13\%), Salticidae $(12.1 \%)$, Theridiidae (11.6\%), Diguetidae y Lycosidae (7.8\% cada una) que hacen un total del $68.6 \%$ del 100\% (Fig. 2). Los géneros más abundantes fueron: Mecaphesa (14.4\%), Psilochorus (11.6\%) y Diguetia (7.7\%) y las especies más abundantes: Latrodectus hesperus Chamberlin \& Ivie, 1935 (33.7\%), Psilocorus imitatus Gertsch \& Mulaik, 1940 (31.2\%) y Diguetia signata Gertsch, 1958 (28.6\%) (Cuadro 1).

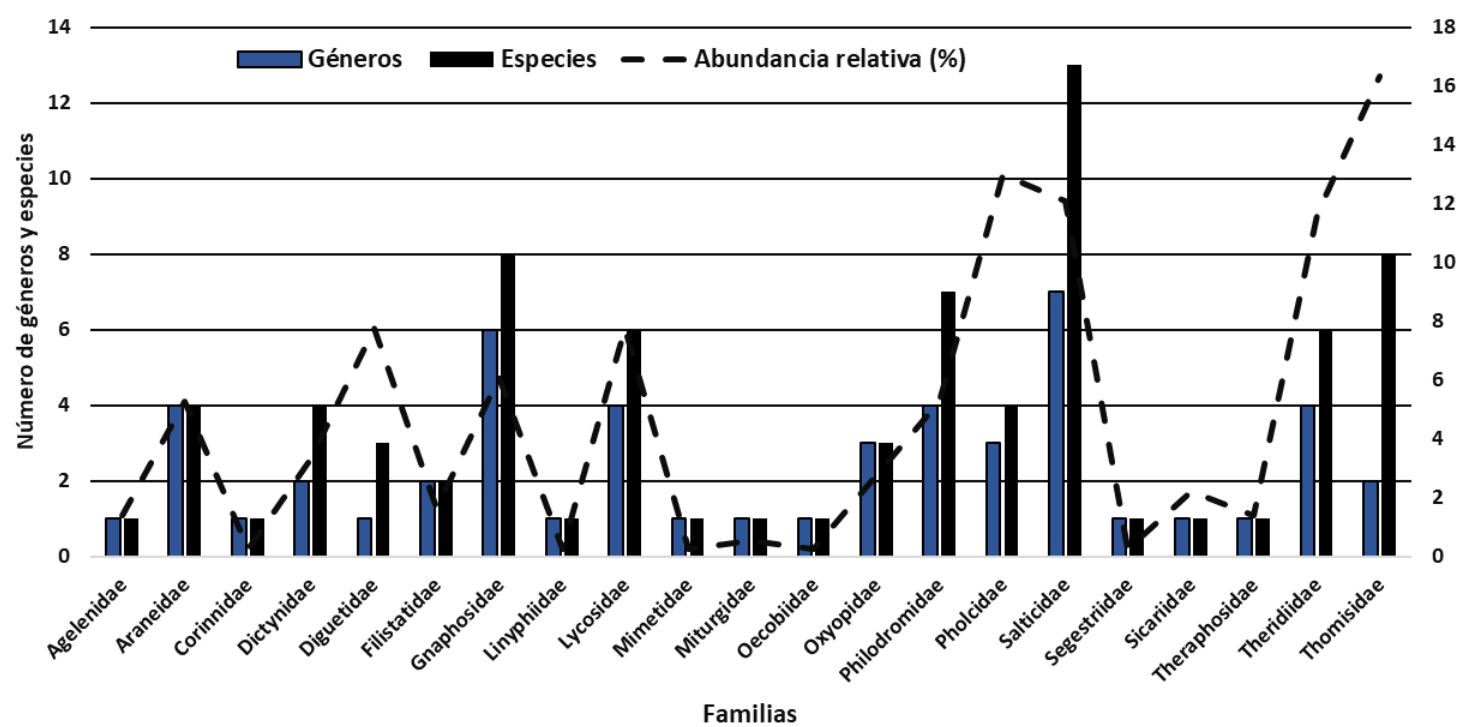

Figura 2. Riqueza y abundancia relativa de géneros de especie por familia de las arañas recolectadas en los Médanos de Samalayuca, Chihuahua. 


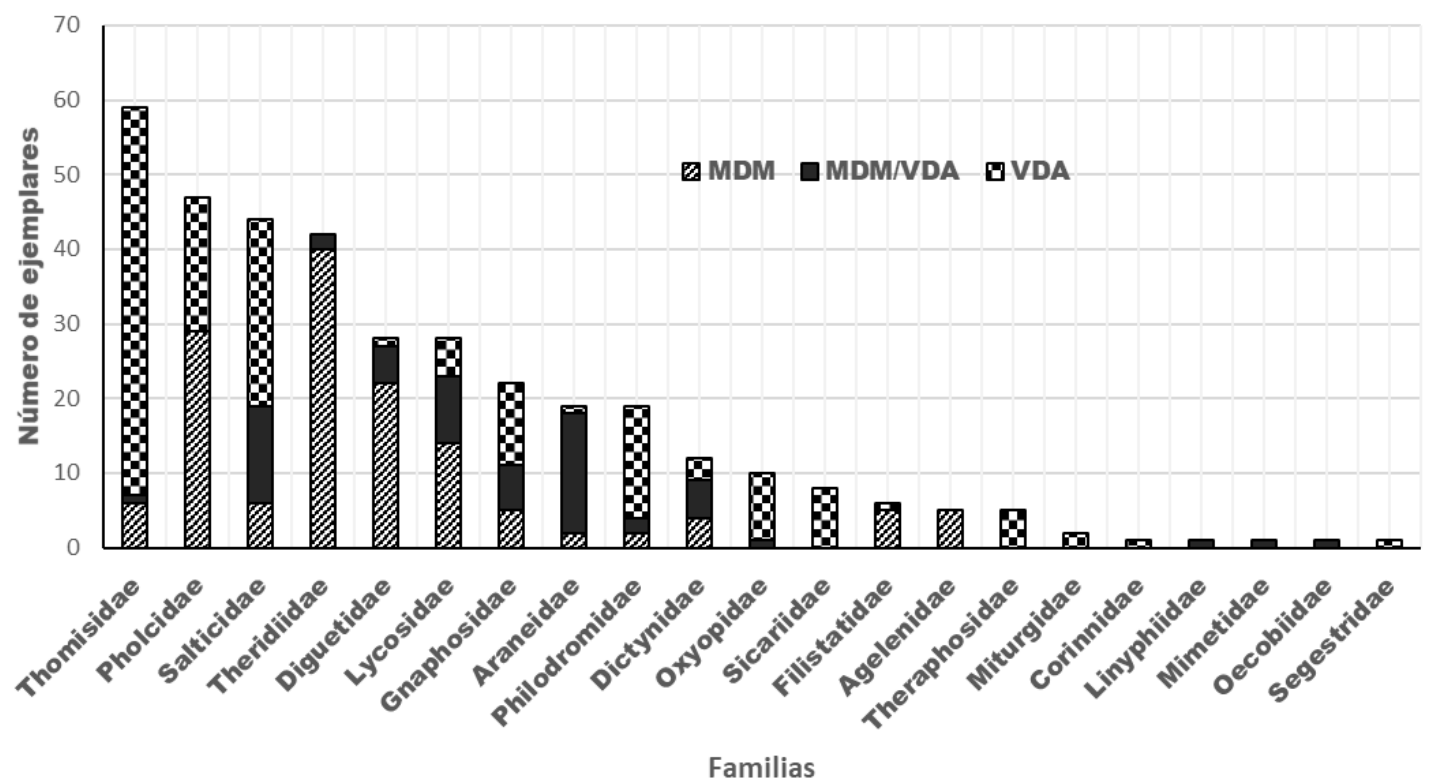

Figura 3. Número de ejemplares por familia de arañas recolectadas en cada tipo de vegetación en los Médanos de Samalayuca, Chihuahua.

Los ejemplares de los géneros Hamataliwa, Steatoda, Ariadna, Phidippus, Syspira, Mimetus y Euryopis no pudieron ser determinados por tratarse de individuos juveniles. En el caso de Philodromus sp. Metaphidippus sp., Filistatoides sp., Emblyna sp., Micaria sp., Hogna sp., Modisimus sp., Mecaphesa sp., solo están representadas por los adultos de un solo sexo, al igual que Emblyna affin. reticulata, E. affin. melva, Sassacus affin. cyaenus, S. affin. samalayuca y Pardosa grupo lapidicina, por lo que para su identificación o validación de las especies se requiere la obtención de ambos sexos y un mayor número de ejemplares. En el caso de Psilochorus sp. y Aphonopelma sp., se debe realizar una revisión más meticulosa de los ejemplares recolectados para su determinación a especie.

En general las arañas del estrato vegetación estuvieron representadas por $55.8 \%$ del total de especies, las arañas del suelo por $40.3 \%$ y las arañas distribuidas en ambos estratos por $3.9 \%$. (Cuadro 1). En cuanto a la zona, MDM agrupó 16 familias, 27 géneros y 31 especies, el ecotono (MDA/VDA) contuvo a 17 familias, 36 géneros y 46 especies y en VDA se registraron 15 familias, 28 géneros y 37 especies (Cuadro 1). Doce familias fueron localizadas en común en todos los tipos de vegetación mientras que Corinnidae, Linyphiidae y Oecobiidae solo tuvieron presencia en el ecotono. Las familias Agelenidae y Segestriidae solo estuvieron presentes en el MDM y la familia Mimetidae se registró solo en la VDA (Fig. 3). De las 77 especies registradas 31 (40.2\%) se localizaron en el MDM, 46 (59.7\%) en el ecotono (MDM/VDA) y 37 (48.1\%) en VDA. El índice de complementariedad entre los estratos fue de $95 \%$ y $84 \%$ entre la comunidad de arañas de la MDM y VDA. El valor estimado de riqueza de especies con el estimador Chao 1, muestra que el esfuerzo de muestreo fue de un $68 \%$ de integridad, quedando 36 especies para ser recolectadas, mientras que con el estimador ACE fue de $73 \%$ de integridad, quedando 27 especies por conocerse (Fig. 4). Ninguno de los estimadores llegó a la asíntota. 


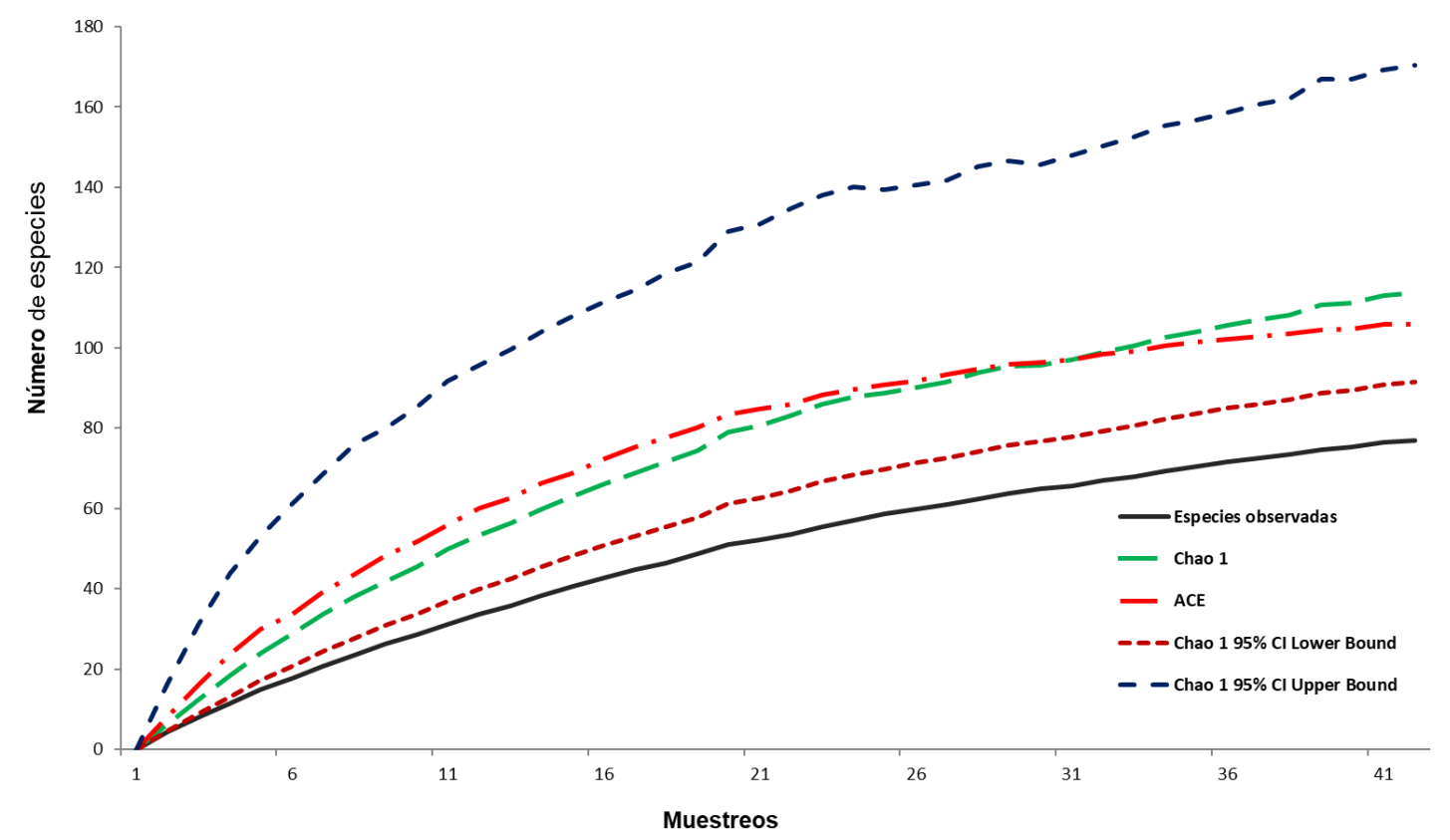

Figura 4. Curva de acumulación de las especies de arañas observadas y estimadas por ACE y Chao1 en los Médanos de Samalayuca, Chihuahua.

\section{Nuevos registros \\ Familia Araneidae, Simon, 1895 \\ Metepeira arizonica Chamberlin \& Ivie, 1942}

(Figs. 5-8)

Material examinado: México, Chihuahua, Médanos de Samalayuca, Sierra Presidio. Altitud 1,370 m. 1 CARCIBAr 4092, 28 junio 2017, 1 + CARCIBAr 4091, 26 junio 2017. 31 $21^{\prime} 38.8^{\prime \prime} \mathrm{N}, 106^{\circ} 22^{\prime} 28^{\prime \prime} \mathrm{O}$. Matorral desértico micrófilo. C. Palacios, I. D. Chávez cols. $1.4 \mathrm{~km} \mathrm{~N}, 6.6 \mathrm{~km}$ O del poblado de Samalayuca.

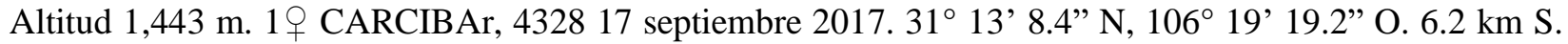
Altitud 1,487 m. 1 q CARCIBAr 4331, 23 septiembre 2017. 31 ${ }^{\circ} 10^{\prime} 15.99 " \mathrm{~N}, 106^{\circ} 16^{\prime}$ '54.58” O. Matorral desértico arenoso. A. García col. $03 \mathrm{Km} \mathrm{S,} 5.5 \mathrm{~km}$ O. Altitud 1,438 m. 1 + CARCIBAr, 4248, 31 julio 2017. $31^{\circ} 12^{\prime} 14.4^{\prime \prime} \mathrm{N}, 106^{\circ} 19^{\prime} 16.96^{\prime}$ ' O. $7 \mathrm{~km} \mathrm{~S}, 5.3 \mathrm{~km}$ O. Altitud 1,433 m. 1 q CARCIBAr 4247, 22 julio 2017. $31^{\circ} 11^{\prime} 2.72^{\prime}$ N, $106^{\circ} 19^{\prime} 15.74$ ” O. Matorral desértico micrófilo. I. D. Chávez col. 4.7 km N, 104.

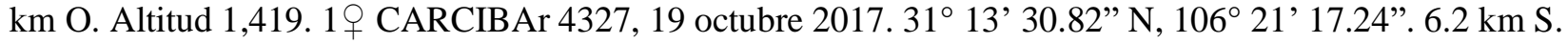
Altitud 1,430 m. 1 q CARCIBAr 4330, 30 septiembre 2017. 31 $10^{\circ}$ ' 15.99” N, 106 $16^{\circ}$ '54.58” O. Matorral

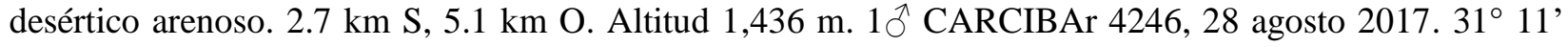
45.6” N, $106^{\circ} 19 ’ 17.54 ”$ O. Matorral desértico micrófilo. A. García col.

Distribución: Estados Unidos y México. En México se cita para Baja California, Coahuila, Durango, San Luis Potosí, Sinaloa, Sonora y Zacatecas (Piel, 2001). Primer registro para Chihuahua.

\section{Familia Corinnidae Castianeira amoena (C. L. Koch, 1842)}

(Figs. 9-10)

Material examinado: México, Chihuahua, Médanos de Samalayuca, Municipio Guadalupe. El Huérfano. Altitud 1,294 m. 1 + CARCIBAr 4096, 19-24 junio 2017. 31 17' 41.5” N, 106³0’ 43" O. Vegetación de matorral desértico. C. Palacios y I. D. Chávez cols. 
Distribución: Estados Unidos y México. De México es citada de Nuevo León (Reiskind, 1969). Primer registro para Chihuahua.
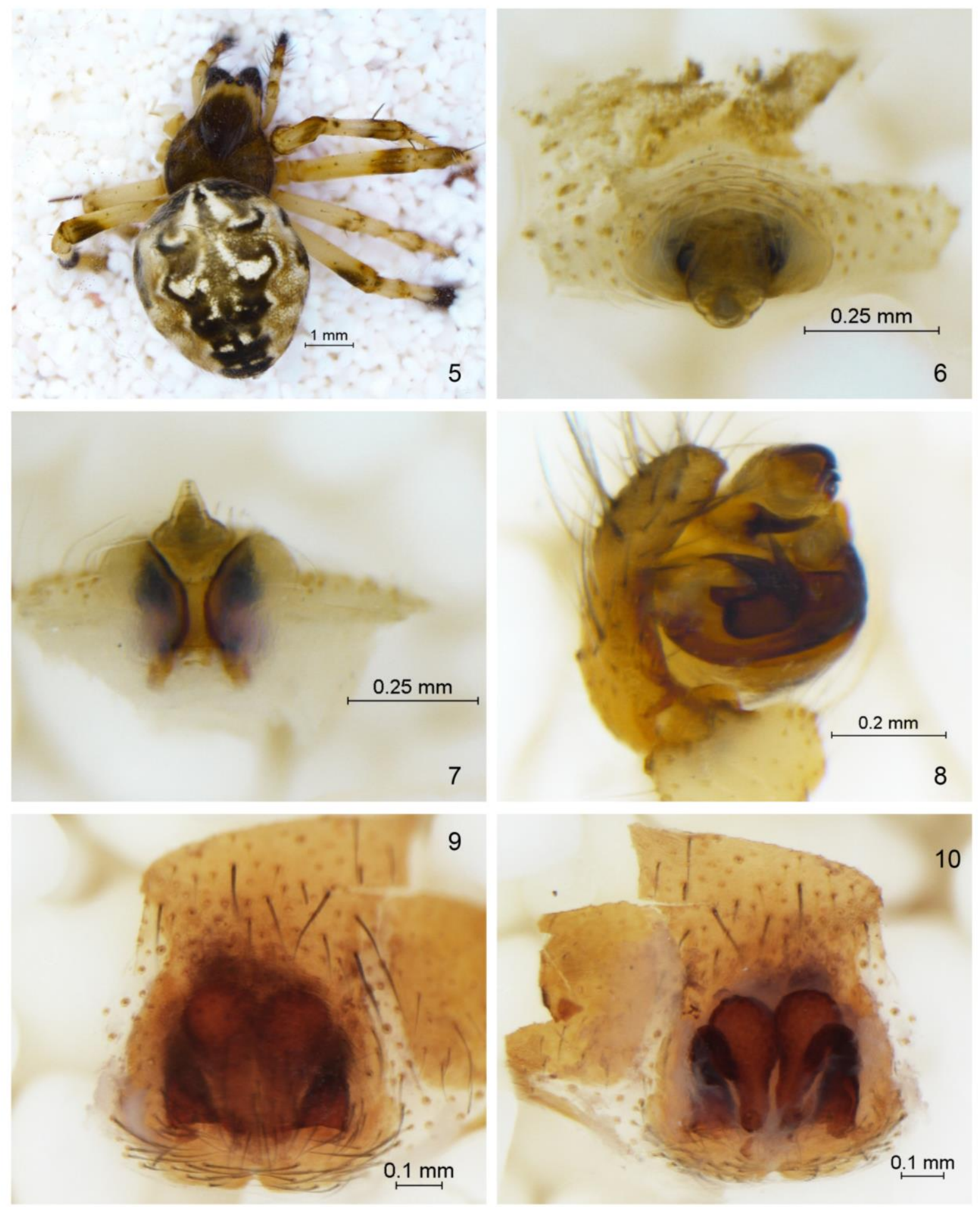

Figuras 5-10. Metepeira arizonica: 5) Hembra vista dorsal, 6) Epigineo vista ventral, 7) Epigineo vista posterior, 8) Pedipalpo vista retrolateral. Castianeira variata: 9) Epigineo vista ventral, 10) Epigineo vista dorsal. 


\section{Familia Dictynidae \\ Dictyna sierra Chamberlin, 1948}

(Figs. 11-12)

Material examinado: México, Chihuahua, Médanos de Samalayuca, Juárez. $4.3 \mathrm{~km} \mathrm{~N}, 6.6 \mathrm{~km}$ O del poblado de Samalayuca. Altitud 1,426 m. $1{ }^{\top}$ CARCIBAr 4339, $1^{\circ}$ octubre 2017. $31^{\circ} 13^{\prime} 27.84$ ' N, $106^{\circ}$ 19'56.24” O. Vegetación de matorral desértico arenoso. I. D. Chávez col.

Distribución: Estados Unidos (Chamberlin \& Gertsch, 1958). Primer registro para México.

\section{Familia Diguetidae \\ Diguetia imperiosa Gertsch \& Mulaik, 1940}

(Figs. 13-15)

Material examinado: México, Chihuahua, Municipio Juárez. $3.5 \mathrm{~km} \mathrm{~S}, 4.4 \mathrm{~km} \mathrm{O}$ del poblado de

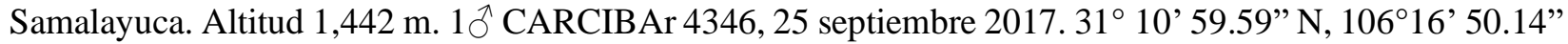

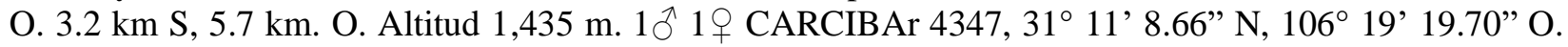
Matorral desértico. A. García col. $3.7 \mathrm{~km} \mathrm{~N}, 6.7 \mathrm{~km} \mathrm{O.} \mathrm{Altitud} \mathrm{1,429.} 19$ CARCIBAr 4344, 10 octubre 2017. $31^{\circ} 13$ ' 21.14” N, 106 19’ 56.49”' O. Matorral desértico. C. Palacios y I. D. Chávez cols.

Distribución: Estados Unidos y México. Para México se cita en Coahuila y Sonora (Gertsch, 1958). Primer registro para Chihuahua.

\section{Familia Gnaphosidae \\ Drassyllus mumai Gertsch \& Riechter, 1976}

(Figs. 16-18)

Material examinado: México, Chihuahua, Médanos de Samalayuca, Municipio Juárez. El Huérfano.

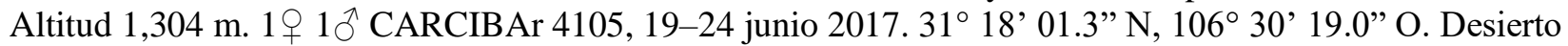

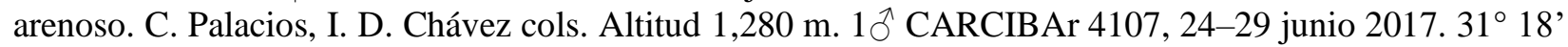
19.6" N, $106^{\circ} 30^{\prime}$ 21.7" O. Matorral desértico micrófilo. C. Palacios, I. D. Chávez cols. Sierra Presidio.

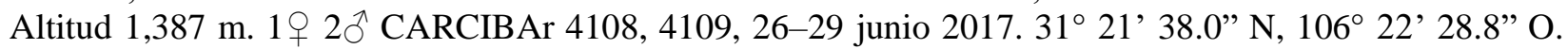

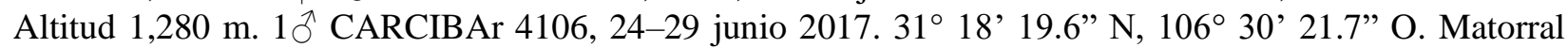
desértico micrófilo. C. Palacios y I. D. Chávez cols.

Distribución: Estados Unidos y México. Para México se cita en Coahuila (Platnick \& Shadab, 1982). Primer registro para Chihuahua.

\section{Herpyllus ecclesiasticus Hentz, 1832}

(Figs. 19-20)

Material examinado: México, Chihuahua, Médanos de Samalayuca, Municipio Juárez. El Huérfano. Altitud 1,317-1,324 m. 2o CARCIBAr 4112 y 4113, 26-29 junio 2017. 31 18 '20" N, 106 31' 25" O, matorral desértico micrófilo. C. Palacios y I.D. Chávez cols. Sierra El Presidio. Altitud 1,430 m. 1ㅇ, 2 juveniles CARCIBAr 4114, 4115 y 4116, 26-29 junio 2017. 31 21 '32.8” $\mathrm{N}, 106^{\circ} 22$ ' 21.9” O. Matorral desértico micrófilo. C. Palacios y I. D. Chávez cols.

Distribución: Canadá, Estados Unidos y México. Para México se cita en Tamaulipas (Platnick \& Shadab, 1977). Primer registro para Chihuahua. 

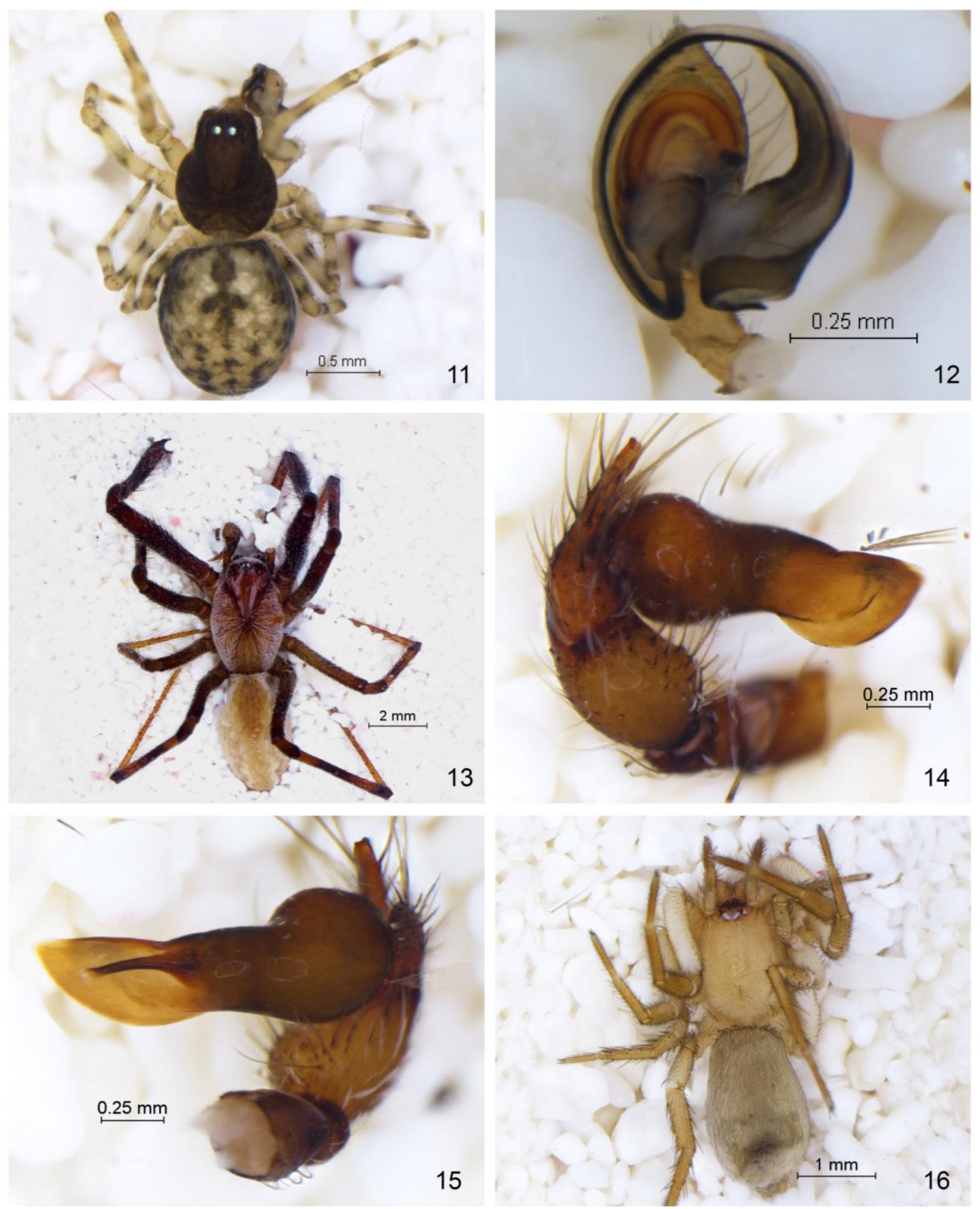

Figuras 11-16. Dictyna sierra: 11) Macho vista dorsal, 12) Pedipalpo vista ventral. Diguetia imperiosa: 13) Macho vista dorsal, 14) Pedipalpo vista retrolateral, 15) Pedipalpo vista prolateral. Drassyllus mumai: 16) Macho vista dorsal. 
Familia Philodromidae Apollophanes texanus Banks, 1904

(Figs. 21-23)

Material examinado: México, Chihuahua, Médanos de Samalayuca, Municipio Juárez, Sierra El Presidio.

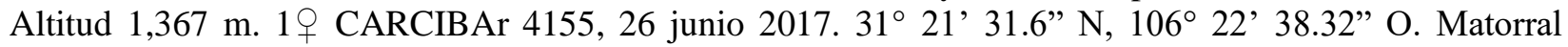
desértico micrófilo. C. Palacios y I. D. Chávez cols. Altitud 1,309 m. 1 q CARCIBAr 4154, 26 junio 2017. $31^{\circ} 18^{\prime} 02.6^{\prime \prime} \mathrm{N}, 106^{\circ} 30^{\prime} 20.6$ O. Desierto arenoso. C. Palacios y I. D. Chávez cols.

Distribución: Estados Unidos y México. Para México se cita en Baja California y Sonora (Dondale \& Redner, 1975). Primer registro para Chihuahua.

\section{Philodromus arizonensis Dondale \& Redner, 1969}

(Figs. 24-26)

Material examinado: México, Chihuahua, Juárez, $3.8 \mathrm{~km} \mathrm{~S}, 5.8 \mathrm{~km}$ O del poblado de Samalayuca. Altitud

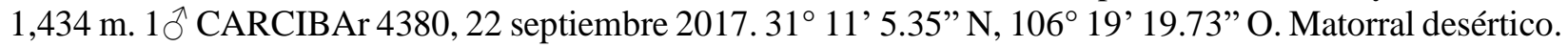

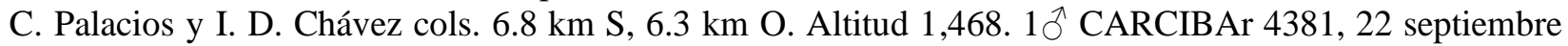
2017. $31^{\circ} 10^{\prime} 51.74 ”$ N, $106^{\circ} 19^{\prime}$ 27.3” O. Matorral desértico. A. García cols.

Distribución: Estados Unidos (Dondale \& Redner, 1969; 1976). Primer registro para México.

\section{Titanebo dondalei (Sauer 1968)}

(Figs. 27-31)

Material examinado: México, Chihuahua, Municipio Juárez. $5.8 \mathrm{~km} \mathrm{~S}, 0.4 \mathrm{~km}$ E del poblado de

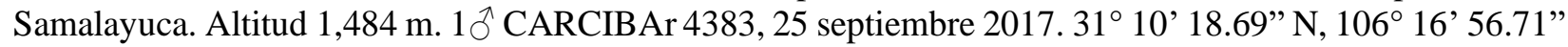
O. $4.3 \mathrm{~km} \mathrm{~N}, 6.6 \mathrm{~km} \mathrm{O}$. Altitud 1,426. 1 + CARCIBAr 4384, 1 octubre 2017. 31 ${ }^{\circ} 13^{\prime} 27.84^{\prime \prime} \mathrm{N}, 106^{\circ} 1^{\prime}$ '

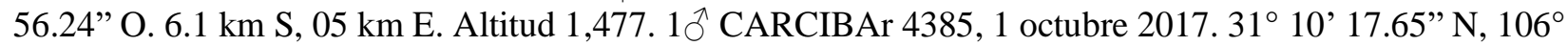
17’ 5.85” O. Matorral desértico arenoso. I. D. Chávez col.

Distribución: Estados Unidos (Sauer, 1968; Sauer \& Platnick, 1972). Primer registro para México.

\section{Familia Pholcidae Psilochorus imitatus Gertsch \& Mulaik, 1940}

(Figs. 32-35)

Material examinado: México, Chihuahua, Municipio Juárez. $13.2 \mathrm{~km} \mathrm{~S}, 2 \mathrm{~km} \mathrm{O}$ del poblado de Samalayuca. Altitud 1,436 m. 1 9,3 juveniles CARCIBAr 4287, 28 junio 2017. $31^{\circ} 7^{\prime}$ '54.00' N, 106 ${ }^{\circ} 7^{\prime}$ 39.43” O. $12.9 \mathrm{~km} \mathrm{~S}, 2.5 \mathrm{~km}$ O. Altitud 1,433m. 10̂, 1 juvenil CARCIBAr 4285, 29 junio 2017. 31 $7^{\circ}$ ' $57.46^{\prime \prime} \mathrm{N}, 106^{\circ} 18^{\prime} 6.29$ ” O. $12.7 \mathrm{~km}$. S, $3.6 \mathrm{~km}$ O. Altitud 1,429 m. 1 ㅇ, 3 juveniles CARCIBAr 4206. $31^{\circ}$ 7' 58.47” N, 106 $17^{\prime} 54.28^{\prime}$ ' S. 6 km S, 0.3 km O. Altitud 1,483 m. 1 q CARCIBAr 4288, 21 agosto 2017.

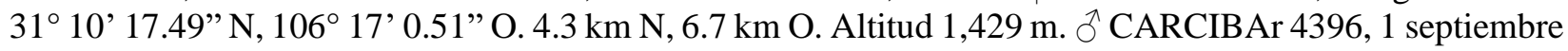
2017. $31^{\circ} 13^{\prime} 27.22^{\prime \prime} \mathrm{N}, 106^{\circ} 19^{\prime} 55.05^{\prime}$ O O. $5.3 \mathrm{~km}$ S. Altitud 1,479. 1 ㅇ CARCIBAr 4397, 24 septiembre 2017. $31^{\circ} 10^{\prime} 26.04^{\prime \prime} \mathrm{N}, 106^{\circ} 17^{\prime} 1.82^{\prime}$ ' O. $2.4 \mathrm{~km}$ O. Altitud 1,439 m. 1 ㅇ 1 $1{ }^{\Uparrow}, 2$ juveniles CARCIBAr 4395, 21 septiembre $2017.31^{\circ} 8^{\prime} 6.14^{\prime \prime} \mathrm{N}, 106^{\circ} 17^{\prime} 45.34^{\prime \prime}$ O. $3.3 \mathrm{~km} \mathrm{~N}, 6.8 \mathrm{~km}$ O. Altitud 1,427. 1 q CARCIBAr 4399,1 octubre $2017.31^{\circ} 13^{\prime} 16.75^{\prime} \mathrm{N}, 106^{\circ} 19^{\prime} 56.20^{\prime}$ O. $4.8 \mathrm{~km} \mathrm{~S}, 5.6 \mathrm{~km}$ O. Altitud 1,428 m. 1 ㅇ CARCIBAr 4398, 1 octubre 2017. $31^{\circ} 10^{\prime} 52.86^{\prime}$ N N, $106^{\circ} 19^{\prime} 32.51$ ” O. Desierto Arenoso. I. D. Chávez col.

Distribución: Estados Unidos (Slowik, 2009). Primer registro para México. Esta especie está registrada por Slowik (2009) para la región Central y Sur de Estados Unidos, sin embargo, tiene un registro en Coahuila, México en su mapa de distribución, que no está mencionado en el texto, posiblemente por ser erróneo dicho registro, por lo que nosotros la consideramos como un primer registro para México. 

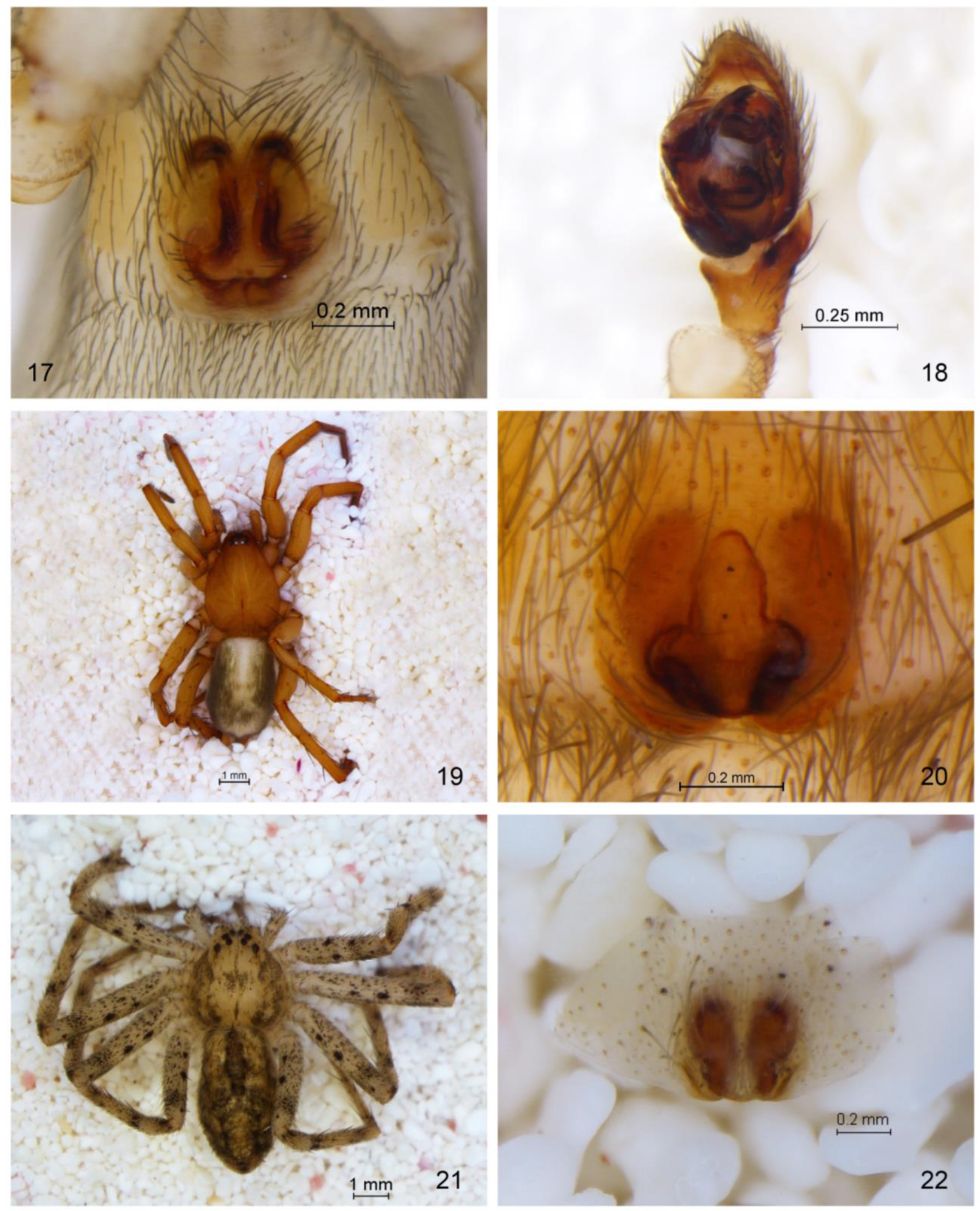

Figuras 17-22. Drassyllus mumai: 17) Epigineo hembra vista ventral, 18) Pedipalpo vista ventral. Herpyllus ecclesiasticus: 19) Hembra vista dorsal, 20) Epigineo vista ventral. Apollophanes texanus: 21) Hembra vista dorsal, 22) Epigineo vista ventral. 

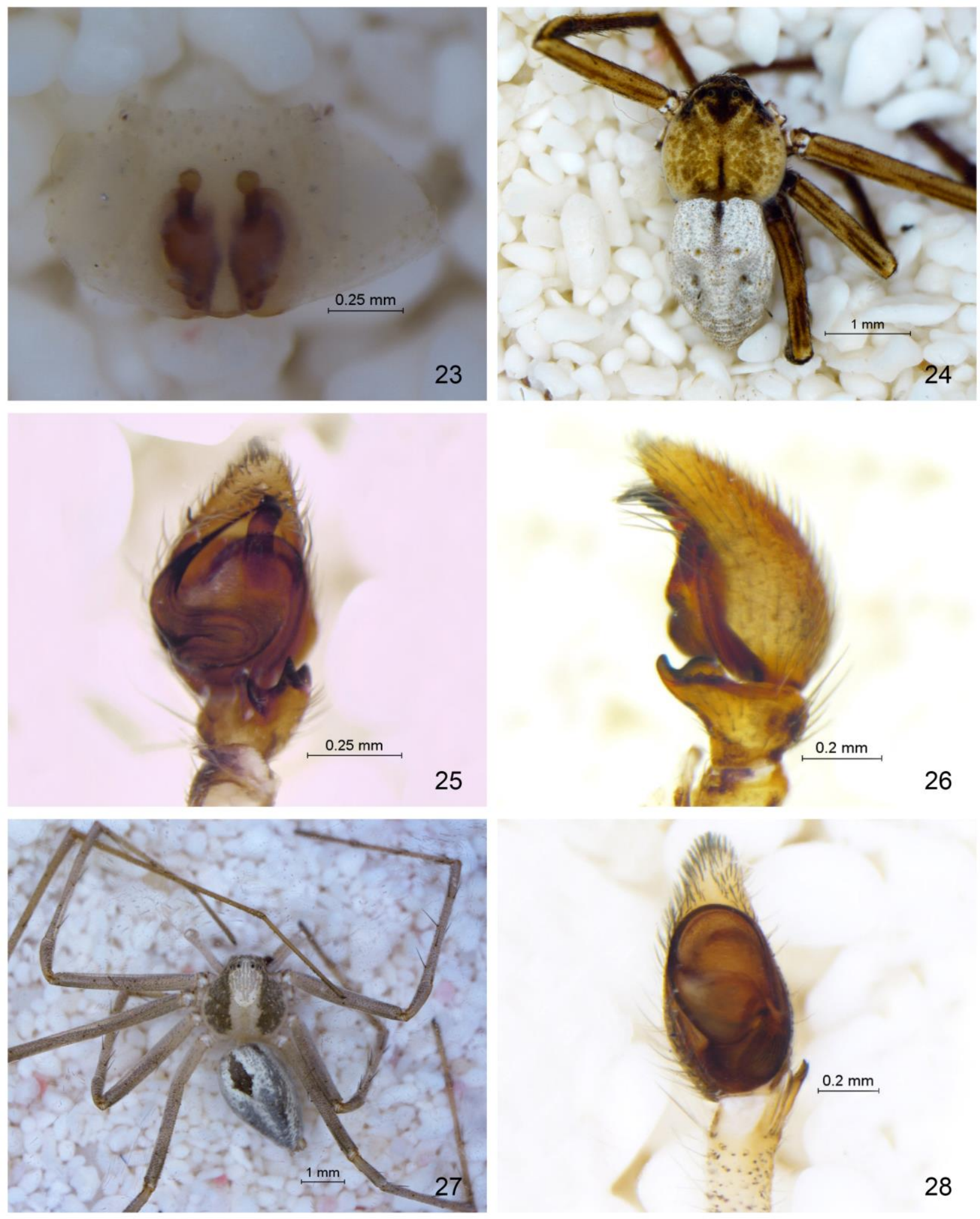

Figura 23-28. Apollophanes texanus: 23) Epigineo vista dorsal. Philodromus arizonensis: 24) Macho vista dorsal, 25) Pedipalpo vista dorsal, 26) Pedipalpo vista retrolateral. Titanebo dondalei: 27) Macho vista dorsal, 28) Pedipalpo vista ventral. 

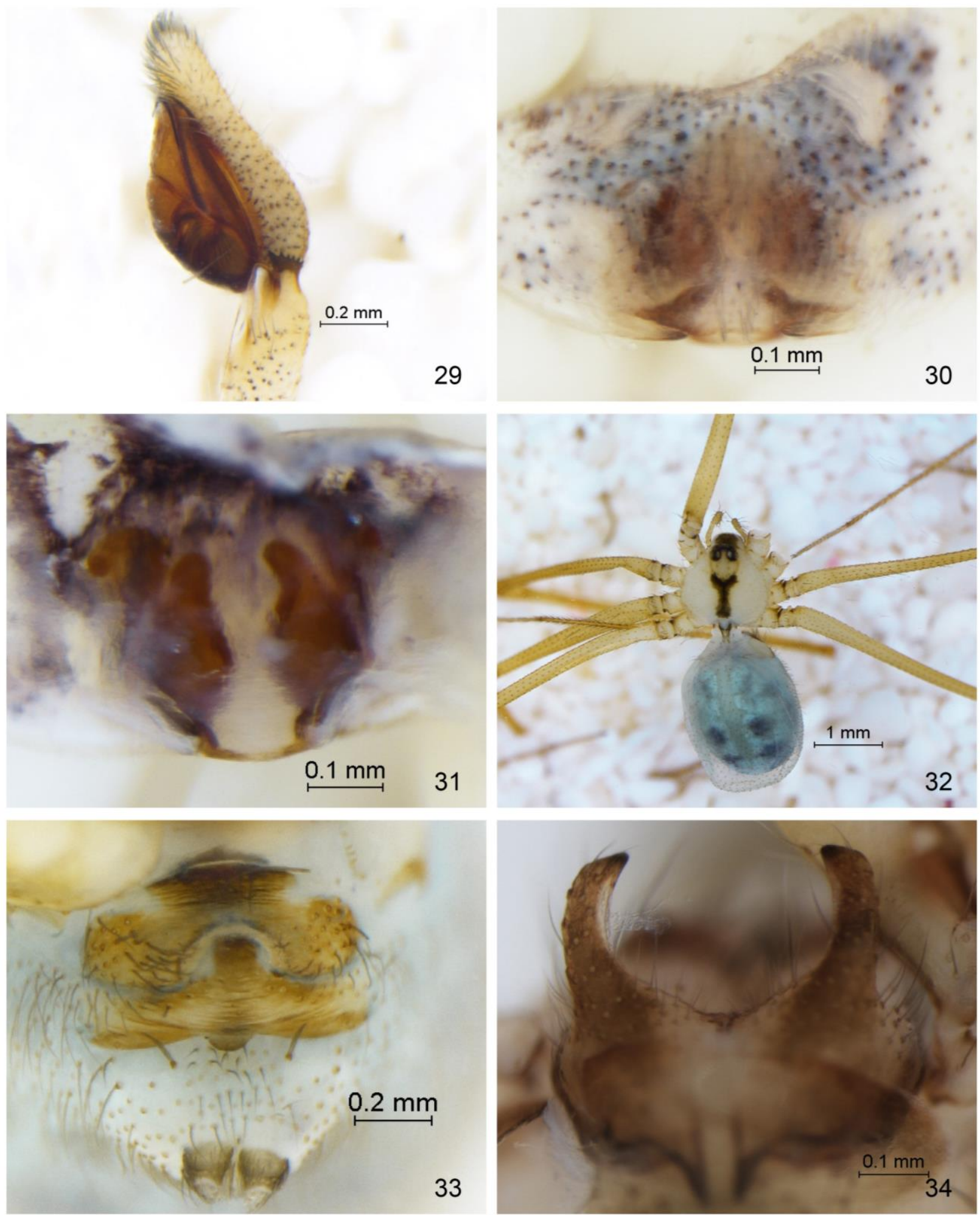

Figuras 29-34. Titanebo dondalei: 29) Pedipalpo vista retrolateral, 30) Epigineo vista ventral, 31) Epigineo vista dorsal. Psilochorus imitatus: 32) Hembra vista dorsal, 33) Epigineo vista ventral, 34) Quelíceros del macho vista frontal. 


\section{Familia Theridiidae \\ Latrodectus hesperus Chamberlin \& Ivie, 1935}

(Figs. 36-37)

Material examinado: México, Chihuahua, Médanos de Samalayuca, Municipio Juárez. 5.8 O poblado de

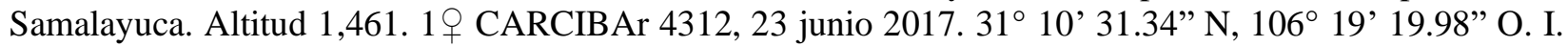
D. Chávez col. $3.7 \mathrm{~km} \mathrm{~N}, 6.8 \mathrm{~km}$ O. Altitud 1,433. 10ิ CARCIBAr 4434, 1 octubre 2017. $31^{\circ} 12^{\prime}$ '56.64"

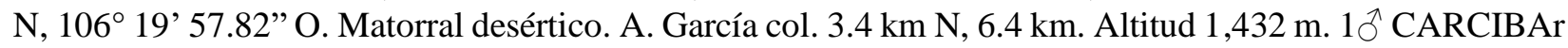
4436, 1 octubre $2017.31^{\circ} 13$ ' $17.65^{\prime}$ N, $106^{\circ} 19^{\prime} 56.02$ " O. I. D. Chávez col. $1.2 \mathrm{~km} \mathrm{~N}, 6.3 \mathrm{~km}$ O. Altitud

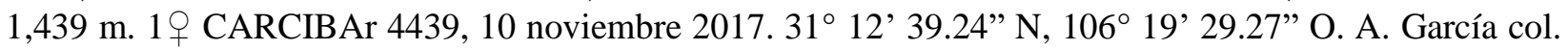

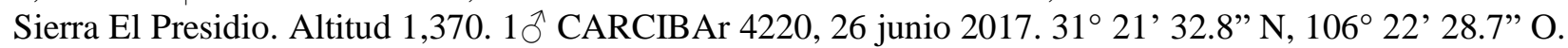

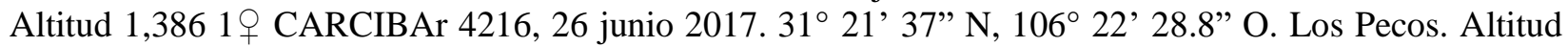

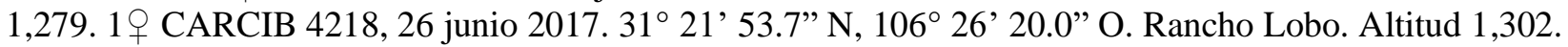
1 ㅇ CARCIBAr 4219, 26 junio 2017. 31 ${ }^{\circ} 13^{\prime} 53.5^{\prime}$ N N, 106 30’ 05” O. Matorral desértico micrófilo. C. Palacios y I. D. Chávez cols.

Distribución: Norteamérica, Israel y Palestina (World Spider Catalog, 2019). México: Baja California Sur (Jiménez et al., 2015) Coahuila (Desales-Lara et al., 2018). Primer registro para Chihuahua.

\section{Familia Thomisidae Mecaphesa coloradensis (Gertsch, 1933) \\ (Figs. 38-41)}

Material examinado: México, Chihuahua, Médanos de Samalayuca, Municipio Guadalupe. El Presidio. Altitud 1,386 m. 1 $\bigcirc^{\Uparrow}, 3$ juveniles CARCIBAr 4237, 26 junio 2017. $31^{\circ} 21^{\prime} 37.7^{\prime \prime} \mathrm{N}, 106^{\circ} 22^{\prime} 28.8^{\prime}$ ' O.

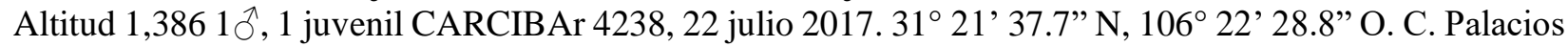
y I. D. Chávez col. $2.4 \mathrm{~km} \mathrm{S,} 6.2 \mathrm{~km}$ O del poblado de Samalayuca. Altitud 1,485 m. $2 \overbrace{}^{\Uparrow} 3$, 3 juveniles CARCIBAr 4317, 22 julio 2017. 31 ${ }^{\circ} 11^{\prime} 30.51^{\prime \prime} \mathrm{N}, 106^{\circ} 19^{\prime} 26.40^{\prime \prime}$ O. Matorral desértico micrófilo. O. I. D. Chávez cols. El Zorro Plateado. Altitud 1,316. 1 q CARCIBAr 4231, 21 junio 2017. 31 17' 18.2” N, $106^{\circ} 28^{\prime} 35.2$ ” O. Matorral desértico arenoso. C. Palacios y I. D. Chávez cols.

Distribución: Estados Unidos y México. Para México se cita en Coahuila (Gertsch, 1939). Primer registro para Chihuahua.

\section{Mecaphesa rothi (Schick, 1965)}

(Figs. 42-44)

Material examinado: México, Chihuahua, Municipio Juárez. $6.3 \mathrm{~km} \mathrm{S,} 14 \mathrm{~km}$ O poblado de Samalayuca Altitud 1,522 m. 10, 3 juveniles CARCIBAr 4321, 26 julio 2017. $31^{\circ}$ 9' $55.57^{\prime \prime} \mathrm{N}, 106^{\circ} 17^{\prime} 27.99^{\prime \prime} \mathrm{O}$. Matorral desértico arenoso. O. I. D. Chávez col.

Distribución: Estados Unidos (Schick, 1965). Primer registro para México. Schick (1965) menciona que Chamberlin (1924) identificó esta especie como M. celer (Hentz, 1847) de la Isla San Ildefonso, Golfo de California, México con cuatro hembras juveniles. Sin embargo, en este trabajo se considera como un nuevo registro para México, porque no existe ninguna figura de esta especie, con la cual pudiéramos comparar.

\section{DISCUSIÓN Y CONCLUSIONES}

Con este trabajo se incrementa en un $47 \%$ la biodiversidad de arañas para el estado de Chihuahua, al aumentar de 100 especies registradas por Hoffmann (1976) a 147 especies. Estos resultados difieren de los registrados por Jiménez (1996) quien reportó 168 especies para esta entidad, sin embargo, no se pudo hacer una comparación porque no proporcionó un listado de especies. 

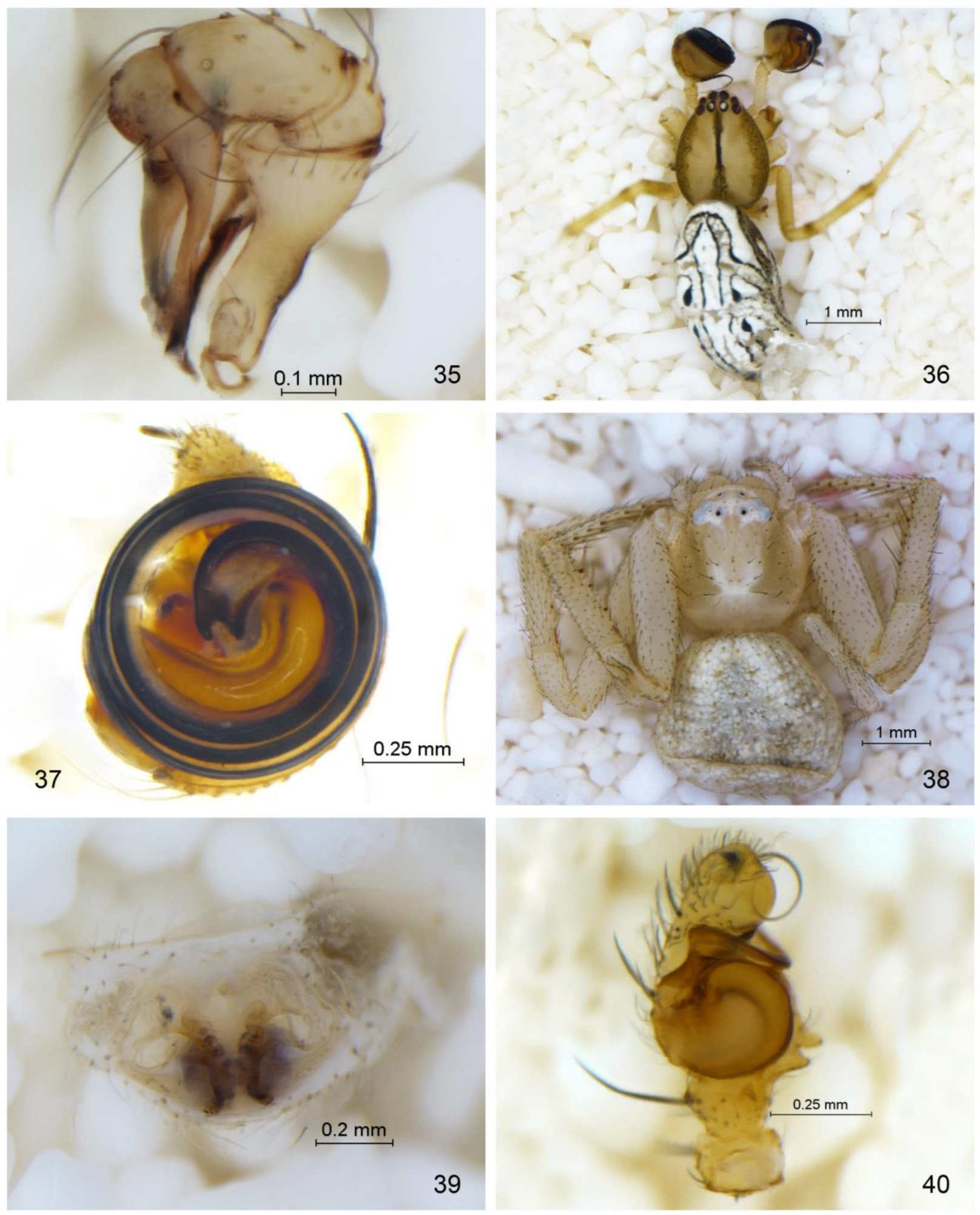

Figura 35-40. Psilochorus imitatus: 35) Pedipalpo del macho vista lateral. Latrodectus hesperus: 36) Macho vista dorsal, 37) Pedipalpo vista ventral. Mecaphesa coloradensis: 38) Hembra vista dorsal, 39) Epigineo vista ventral. 

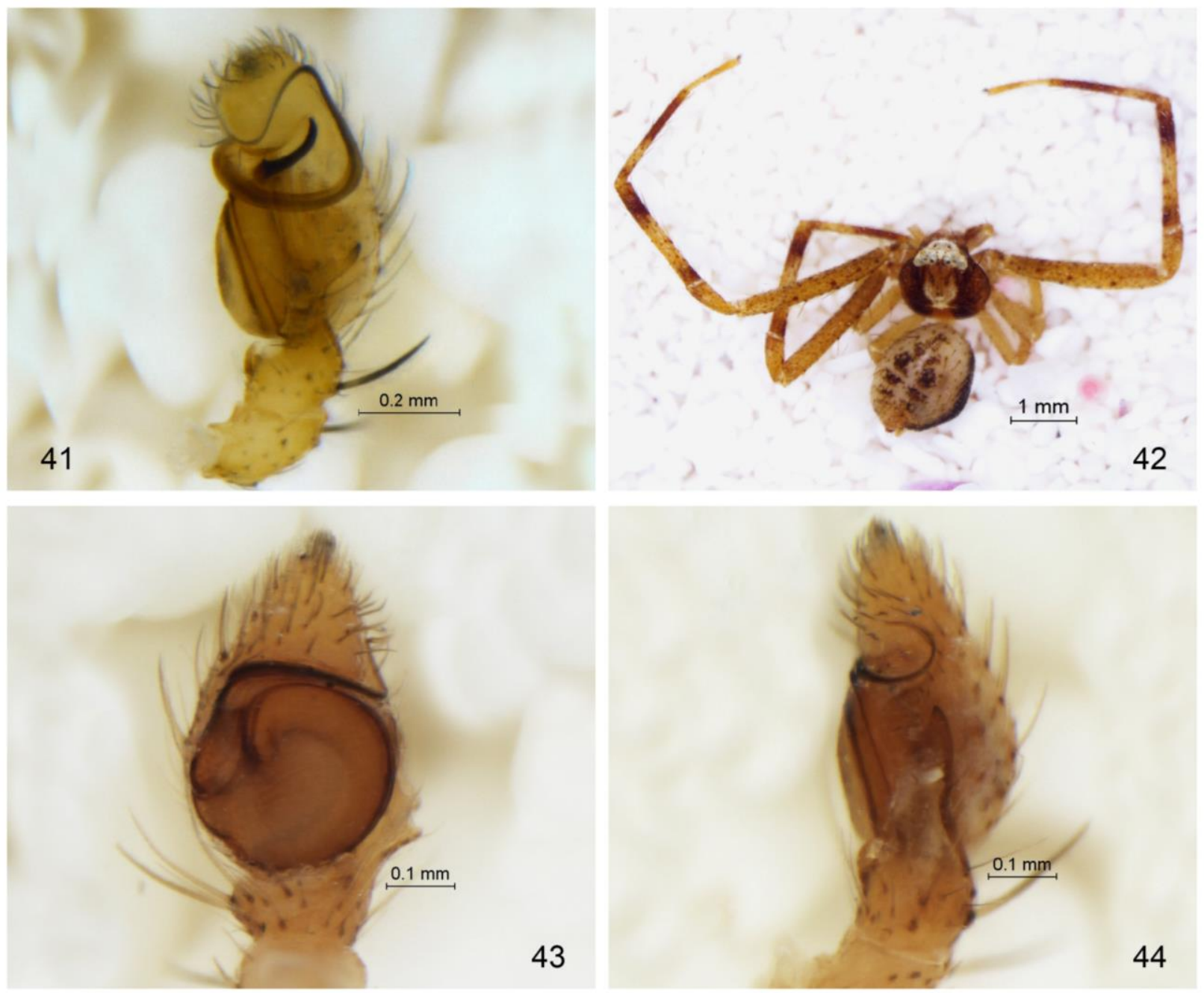

Figuras 40-44. Mecaphesa coloradensis: 40) Pedipalpo vista dorsal, 41) Pedipalpo vista retrolateral. Mecaphesa rothi: 42) Macho vista dorsal, 43) Pedipalpo vista ventral, 44) Pedipalpo vista retrolateral.

Los nuevos registros encontrados para México en este estudio han sido previamente documentados para Estados Unidos y Canadá, y los nuevos registros para Chihuahua han sido citados para Baja California, Baja California Sur, Coahuila, Durango, San Luis Potosí, Sinaloa, Sonora, Zacatecas, Nuevo León y Tamaulipas, lo que demuestra que en conjunto tienen una gran afinidad a las zonas áridas de Norteamérica. Al comparar nuestros resultados con otros trabajos sobre la diversidad de arañas en los desiertos de América del Norte, observamos que el número de familias, géneros y especies fue mayor que los encontrados por Chew (1961) en el matorral desértico en Arizona, donde registró 4 familias, 11 géneros y 13 especies de arañas asociadas a L. divaricata; Muma (1975) reportó 11 familias, 19 géneros y 23 especies de arañas del suelo en Tularose, Nuevo México; de igual forma Hatley y MacMahon (1980) registraron un menor número de familias (11) y especies (40) en el cañón Verde en Cache Country en Utah, mientras que Broussard y Horner (2006) reportaron 24 familias, 46 géneros y 66 especies de arañas del suelo en Trans-Pecos y El Presidio al oeste de Texas. En México Jiménez y Navarrete (2010) observaron de igual manera un mayor número de familias (22), géneros (44) y especies (52) de arañas del suelo en el matorral sarcocaule en Baja California Sur. Probablemente las diferencias encontradas en la riqueza de especies de este estudio y las localidades desérticas antes mencionadas, sea porque se incluyeron especies de suelo y vegetación, mientras que en los trabajos anteriores solo consideraron a las arañas de un solo estrato. 
En trabajos posteriores donde también incluyeron uno o ambos estratos, se destinó un mayor tiempo de recolecta, por lo que la riqueza de arañas fue superior a las encontrada por nosotros, como en el caso de Lightfoot et al. (2008), quienes reportaron 18 familias y 121 especies de arañas del suelo en cinco ecorregiones desérticas de Nuevo México. De igual forma, Richman et al. (2011) registraron una mayor riqueza (33 familias y 231 especies) de arañas debido a un intenso muestreo de 30 años en la vegetación desértica de cuatro áreas del desierto Chihuahuense. Por su parte, Jiménez et al. (2015) también proporcionó mayor número de familias (34), géneros (130) y especies (243) de los oasis y áreas circundantes de la región meridional la península de Baja California en un periodo de tres años y Corcuera et al. (2019) registró 37 familias y 159 especies de arañas en diversos tipos de vegetación de El Churince, Cuatro Ciénegas, Coahuila capturadas durante cinco años de recolecta y en diversos períodos del año.

De acuerdo con los criterios de Corcuera et al. (2019), estas diferencias de nuestro inventario con otras zonas desérticas pueden obedecer a varios factores, como la duración de los periodos de muestreo, los métodos empleados en la captura, la complejidad de la estructura de vegetación y el esfuerzo de recolecta. De esta manera el tamaño y composición de las especies de arañas de un lugar determinado variará con el tiempo, y a medida que se prolongue el muestreo o que se añada un mayor número de muestras en el mismo lapso, aparecerán más especies raras que enriquecerán el inventario (Jiménez-Valverde \& Hortal, 2003; Corcuera et al., 2019). En nuestro caso, la abundancia de arañas muestra que el esfuerzo de muestreo fue insuficiente, como lo indica la curva de acumulación de especies, misma que no llegó a la asíntota (Fig. 4). No obstante, Cardoso et al. (2007) indica que no es necesario realizar un muestreo del 90-100\% en estudios sobre artrópodos, porque el esfuerzo de muestreo puede ser representativo con un inventario menos extenso (70 a 80\%); sin embargo, consideramos que nuestro inventario aún no está completo y que es necesario mejorar los métodos de captura y realizar un mayor esfuerzo de recolecta y en otras épocas del año.

Por otro lado, si comparamos el número total de especies de este trabajo con el número total de especies de las localidades más diversas en los desiertos de Norteamérica (Cuadro 2), observamos que tenemos un 33\% de las especies para Jornada del Muerto, White Sands, Valley of Fire y Big Bend, un 48\% de las especies de Cuatro Ciénegas (ambas en el desierto Chihuahuense) y un 31\% de las especies registradas para los oasis de Baja California Sur (desierto Sonorense), así como 36, 12 y 13 especies en común respectivamente. Entre estas especies destacan Oxyopes tridens Brady, 1964 y Apollophanes texanus Banks, 1904 seguidas de Zelotes lasalanus Chamberlin, 1928, Habronattus conjunctus (Banks, 1898) y Mecaphesa coloradensis (Gertsch, 1933) (Cuadro 1) con amplia distribución en las zonas áridas en Norteamérica. Al respecto es conveniente mencionar que aun cuando L. hesperus, $P$. imitatus y D. signata fueron las especies más abundantes y mayormente distribuidas en este trabajo, su presencia no ha sido suficientemente documentada en nuestro país. Sin embargo, con excepción de D. signata y P. imitatus limitadas al sur de Estados Unidos (Gertsch, 1958) y a la región central de ese país (Slowick, 2009), L. hesperus tiene una amplia distribución desde Canadá, Estados Unidos y algunas localidades en México. También ha sido registrada de Israel y Palestina (Levy \& Amitai, 1983) donde posiblemente ha sido introducida.

Como era de esperarse, las familias Salticidae y Gnaphosidae como cazadoras errantes, fueron las que agruparon los mayores números de géneros (7 y 6, respectivamente) y especies (13 y 8, respectivamente), pues ambas están bien representadas en las zonas desérticas a nivel mundial (Polis \& Yamashita, 1991). Estas familias también han sido registradas como muy ricas en suelos de comunidades áridas de Nuevo México (Muma, 1975; Lightfoot et al., 2008), en la vegetación desértica de Texas (Broussard \& Horner, 2006; Richman et al., 2011), en una localidad del matorral sarcocaule al sur de la península de Baja California (Jiménez \& Navarrete, 2010) y en los oasis de esa misma región (Jiménez et al., 2015), así como en Cuatro Ciénegas, Coahuila y en el estado de Coahuila (Bizuet et al., 2015; DesalesLara et al., 2018; Corcuera et al., 2019). 
Cuadro 2. Riqueza de especies de arañas en localidades de los desiertos de Norteamérica (Modificado de BizuetFlores, com. pers.)

\begin{tabular}{llccc}
\hline Localidades & Citas bibliográficas & Familia & Especies & Desiertos \\
\hline Médanos de Samalayuca & Este trabajo & 21 & 77 & Chihuahuense \\
\hline Trans Pecos y Presidio Texas & Broussard \& Horner, 2006 & 24 & 66 & Chihuahuense \\
\hline $\begin{array}{l}\text { Jornada del Muerto, White Sands, } \\
\text { Valley of Fire, Big Bend }\end{array}$ & Richman et al., 2011 & 33 & 231 & Chihuahuense \\
\hline Cuatro Ciénegas & Corcuera et al., 2019 & 37 & 159 & Chihuahuense \\
\hline Cañón Verde Cache Country & Hatley \& MacMahon, 1980 & 11 & 40 & Meseta del Colorado \\
\hline Portal Cochise Country & Chew, 1961 & 4 & 13 & Sonorense \\
\hline Tularouse Nuevo México & Muma, 1975 & 11 & 23 & Sonorense \\
\hline Nuevo México & Lightfoot et al., 2008 & 18 & 121 & Sonorense \\
\hline Comitán, B.C.S. & Jiménez \& Navarrete, 2010 & 22 & 52 & Sonorense \\
\hline Oasis Baja California Sur & Jiménez et al., 2015 & 34 & 243 & Sonorense \\
\hline
\end{tabular}

Por otra parte, en el estrato de vegetación se encontró el mayor número de familias (13) y especies (43) debido a la alta riqueza de especies de Thomisidae (7), Philodromidae (7) y Salticidae (13), en el estrato del suelo el número de familias y especies fue menor con 11 y 31, respectivamente, siendo las familias mejor representadas Gnaphosidae (8), Lycosidae (6) y Theridiidae (6). En cuanto al índice de complementariedad entre los estratos fue similar al obtenido por Jiménez et al. (2015) (92.2\%) para los oasis de Baja California Sur. Esto demuestra que ambos estratos comparten muy pocas especies, teniendo un alto grado de especificidad en el hábitat donde las condiciones ambientales son muy diferentes. Los valores de complementariedad (84\%) de la comunidad de arañas de la MDM y VDA también indican que estas comunidades son distintas. La familia Mimetidae y 15 especies más de varias familias solo se circunscriben a VDA (Cuadro 1). Esta comunidad vegetal agrupa plantas de talla pequeña, muy escasas y dispersas como el chamizo (Atieplex canescens) estafiate (Artemisa filifolia) y mezquite (Prosopis juliflora), con sitios completamente carentes de vegetación y con montículos de arena y dunas que son movidas constantemente por el viento (CONANP, 2013). Aquí, las arañas probablemente están mejor adaptadas a las condiciones secas y casi sin vegetación, donde especies como $H$. conjunctus, L. hesperus y $M$. coloradensis son dominantes. En cambio, el MDM se caracteriza por presentar especies vegetales arbustivas de mediano tamaño como la gobernadora (L. tridentata), hojasén (Fluorencia cermia), mariola (Parthenium incanum), escasos mesquites ( $P$. juliflora) y largoncillo (Acacia constricta) (CONANP, 2013). En esta vegetación las familias Lycosidae, Pholcidae estuvieron mejor representadas y Agelenidae y Segestriidae solo aquí fueron registradas, mientras que Mecaphesa sp, L. apachea y $S$. variata fueron las especies más abundantes, debido a que la estructura vegetal fue más compleja, variada y que pudo ofrecer mayor protección y variedad de microambientes disponibles para su establecimiento.

Las 46 especies compartidas en el ecotono (MDM/VDA) y en ambos tipos de vegetación (MDM y VDA) (Cuadro 1), pudieran considerarse como especies más adaptables y con mayor tolerancia ambiental a las variaciones climáticas extremas del desierto, que les permiten transitar o permanecer en este medio, sugiriendo que la distribución de las arañas registradas en este trabajo está asociada a las características florísticas y estructurales de la vegetación.

AgradeCimientos. A Ana Bertha Gatica Colima (UACJ) por la organización de las expediciones y apoyo financiero para el campo, al Laboratorio de Ecología Pesquera por el apoyo en la toma de fotografías al microscopio, A Gerardo Hernández para la edición de fotografías. En especial, al editor asociado Guillermo Ibarra-Núñez por sus atinados comentarios y a los revisores anónimos por sus correcciones y sugerencias al manuscrito. Este trabajo fue realizado como colaboración en el proyecto: Inventario multitaxonómico del ANP Médanos de Samalayuca (PJ018 CONABIO). Permiso de colecta No. SGPA/DGVS/003239/18. 


\section{LITERATURA CITADA}

Álvarez, G. A. (1973) Boletín Meteorológico: Compendio de 1957 a 1971, Estado de Chihuahua, Unión Ganadera Regional de Chihuahua. Boletín, 10, 153-157.

Anderson, S. (1972) Mammals of Chihuahua Taxonomy and distribution. Bulletin of the American Museum of Natural History, 148 (2), 151-410.

Bizuet-Flores, M. Y., Jiménez-Jiménez, M. L., Zavala-Hurtado, A., Corcuera, P. (2015) Diversity patterns of ground dwelling spiders (Arachnida: Araneae) in five prevailing plant communities of the Cuatro Ciénegas Basin, Coahuila, Mexico. Revista Mexicana de Biodiversidad, 86, 153-163. https://doi.org/10.7550/rmb.45444

Broussard, G. H., Horner, N. V. (2006) Cursorial spiders (Arachnida: Araneae) in the Chihuahuan desert of Western Texas, U.S.A. Entomological News, 117 (3), 249-260. https://doi.org/10.3157/0013-872X(2006)117[249:CSAAIT]2.0.CO;2

Cardoso, P., Henriques, S. S., Gaspar, C., Crespo, L. C., Carvallo, R., Schmidt, J. B., Sousa, P., Szüts, T. (2007) Species richness and composition assessment of spiders in a Mediterranean scrubland. Journal of Insect Conservation, 13, 45-55. https://doi.org/10.1007/s10841-007-9116-3

Cepeda, J., Whitford, W. G. (1990) Microartrópodos edáficos del desierto Chihuahuense, al norte de México. Folia Entomológica Mexicana, 78, 257-272.

Chamberlin, R. V. (1924) The spider fauna of the shores and islands of the Gulf of California. Proceedings of the California Academy of Sciences, 12, 561-694.

Chamberlin, R. V., Gertsch, W. J. (1958) The family Dictynidae in America north of Mexico. Bulletin of the American Museum of Natural History, 116, 1-152.

Chew, R. M. (1961) Ecology of the spiders of a Desert community. Journal New York Entomological Society, 69 (1), 5-41.

Colwell, R. K. (2013) EstimateS: Statistical Estimation of Species, Richness and shared species from simples (Software y Guía de usuario). Versión 9.1. Disponible en: http:/Viceroy.colorado.edu/estimates/EstimateSPages/EstimateSRegistration.htm

Colwell, R. K., Coddington, J. A. (1994) Estimating terrestrial biodiversity through extrapolation. Philosophical Transactions of the Royal Society of London Series B, 34, 101-118. https://doi.org/10.1098/rstb.1994.0091

CONANP (Comisión Nacional de Áreas Naturales Protegidas) (2013) Programa de Manejo del Área de Protección de Flora y Fauna Médanos de Samalayuca. 1ª. Edición. SEMARNAT, México, 169 pp.

Corcuera, P., Jiménez, M. L., Desales-Lara, M. A. (2019) The spiders of the Churince region, Cuatro Cienegas Basin: A comparison with Other Desert Areas of North America. Pp: 61-75. In: F. Alvarez, M. Ojeda (Eds.). Animal diversity and Biogeography of Cuatro Cienega's Basin: An endangered hyperdiverse oasis. Springer Nature Switzerland, A.C., Switzerland. https://doi.org/10.1007/978-3-030-11262-2_5

Desales-Lara, M. A., Jiménez, M. L., Corcuera, P. (2018) Nuevos registros de arañas (Arachnida: Araneae) para México y listado actualizado de la araneofauna del Estado de Coahuila. Acta Zoológica Mexicana (nueva serie), 34 (1), 50-63. http://dx.doi.org/10.21829/azm.2018.3411183

Dondale, C. D., Redner, J. H. (1969) The infuscatus and dispar groups of the spider genus Philodromus in North and Central America and the West Indies (Araneida: Thomisidae). The Canadian Entomologist, 101 (9), 921-954. https://doi.org/10.4039/Ent101921-9

Dondale, C. D., Redner, J. H. (1975) Revision of the spider genus Apollophanes (Araneida: Thomisidae). The Canadian Entomologist, 107 (11), 1175-1192. https://doi:10.4039/Ent1071175-11

Dondale, C. D., Redner, J. H. (1976) A review of the spider genus Philodromus in the Americas (Araneida: Philodromidae). The Canadian Entomologist, 108 (2), 127-157. 
https://doi.org/10.4039/Ent108127-2

Gertsch, W. J. (1939) A revision of the typical crab spider (Misumeninae) of America North of Mexico. Bulletin of the American Museum of Natural History, 76, 277-442.

Gertsch, W. J. (1958) The spider family Diguetidae. American Museum Novitates, 1904, 1-24.

Hoffman, A. (1976) Relación bibliográfica preliminar de las arañas de México. (Arachnida:Araneae). Publicaciones especiales Instituto de Biología, Universidad Autónoma de México, UNAM, México. 3, 166-174.

Hatley, C. D., MacMahon, J. A. (1980) Spider community Organization: Seasonal Variation and the Role of Vegetation Architecture. Environmental Entomology, 9 (5), 632-639. https://doi.org/10.1093/ee/9.5.632

Ibarra-Núñez, G. (2014) Arañas como bioindicadores. Pp: 273-290. In: C. A. González-Zuarth, A. Vallarino, J. C. Pérez-Jiménez, A. M. Low-Pfeng (Eds.). Bioindicadores: Guardianes de nuestro futuro ambiental. Colegio de la Frontera Sur (ECOSUR) e Instituto Nacional de Ecología y Cambio Climático (INECC).

Jiménez, M. L. (1996) Araneae. Pp: 83-101. In: J. Llorente, A. N. García-Aldrete, E. González-Soriano (Eds.). Biodiversidad, taxonomía y biogeografía de artrópodos de México: hacia una síntesis de su conocimiento. Universidad Nacional Autónoma de México, México, D.F.

Jiménez, M. L., Navarrete, J. G. (2010) Fauna de arañas de suelo de una comunidad árido-tropical en Baja California Sur, México. Revista Mexicana de Biodiversidad, 81, 417-426.

Jiménez, M. L., Nieto-Castañeda, I. G., Correa-Ramírez, M. M., Palacios-Cardiel, C. (2015) Las arañas de los oasis de la región meridional de la península de Baja California, México. Revista Mexicana de Biodiversidad, 86, 319-331.

https://doi.org/10.1016/j.rmb.2015.04.028

Jiménez-Valverde, A., Hortal, J. (2003) Las curvas de acumulación de especies y la necesidad de evaluar la calidad de los inventarios biológicos. Revista Ibérica de Aracnología, 8, 151-161.

Lemos-Espinal, J. A., Smith, G. R., Ballinger, R. E., Smith, H. M. (2003) Ecology of Sceloporus undulatus speari (Sauria: Phrynosomatidae) from North. Central Chihuahua, México. Journal of Herpetology, 37 (4), 722-725. https://doi.org/10.1670/206-02A

Levy, G., Amitai, P. (1983) Revision of the widow-spider genus Latrodectus (Araneae: Theridiidae) in Israel. Zoological Journal of the Linnean Society, 77 (1), 39-63. https://doi.org/10.1111/j.1096-3642.1983.tb01720.x

Lightfoot, D. C., Brantley, S. L., Allen, C. D. (2008) Geographic patterns of ground-dwelling arthropods across an ecoregional transition in the North American Southwest. Western North America Naturalist, 68 (1), 83-102. https://doi.org/10.3398/1527-0904(2008)68[83: GPOGAA]2.0.CO;2

MacKay, W. P., MacKay, E. E., Perez-Dominguez, J. F., Sánchez, L. V., Vielma-Orozco, P. (1985) Las hormigas del estado de Chihuahua México: el género Pogonomyrmex (Hymenoptera: Formicidae). Sociobiology, 11 (1), 39-54.

Muma, M. H. (1975) Long term can trap for population analyses of ground-surface, Arid-Land arachnids. The Florida Entomologist, 58 (4), 257-270. https://doi.org/10.2307/3493687

Ontiveros, I. S. (2011) Avifauna de Ojo de la Punta, Samalayuca, Juárez, Chihuahua. Tesis de Licenciatura en Biología, Universidad Autónoma de Ciudad Juárez, México.

Piel, W. H. (2001) The systematics of Neotropical orb-weaving spiders in the genus Metepeira (Araneae:Araneidae). Bulletin of the Museum of Comparative Zoology, 157 (1), 1-92.

Platnick, N. I., Shadab, M. U. (1977) A revision of the spider Herpyllus and Scotophaeus (Araneae, Gnaphosidae) in North America. Bulletin of the American Museum of Natural History, 159 (1), 149.

Platnick, N. I., Shadab, M. U. (1982) A revision of the American spiders of the genus Drassyllus (Araneae,Gnaphosidae). Bulletin of the American Museum of Natural History, 173 (1), 1-97. 
Polis, G. A., Hurd, S. D. (1995) Extraordinary high spider densities on islands flew of energy from the marine to terrestrial food webs and the absence of predation. PNAS, 10, 4382-4386. https://doi.org/10.1073/pnas.92.10.4382

Polis, G. A., McCormick, S. J. (1986) Scorpions, spiders and solpugids: predation and competition among distantly related taxa. Oecologia, 71 (1), 111-116.

Polis, G. A., Yamashita, T. (1991) The ecology and importance of predaceous arthropods in desert communities. Pp: 180-222. In: G. A. Polis (Ed.). The ecology of desert communities. University of Arizona Press.

Reiskind, J. (1969) The spider subfamily Castianeirinae of North and Central America (Araneae, Clubionidae). Bulletin of Museum Comparative Zoology, 138, 163-325.

Richman, D. B. (2008) Revision of the jumping spider genus Sassacus (Araneae, Salticidae, Dendryphantinae) in North America. The Journal of Arachnology, 36 (1), $26-48$. https://doi.org/10.1636/H07-03.1

Richman, D. B., Brantley, S. L., Hu, D. H-C., Whitehouse, M. E. A. (2011) Spiders of the Chihuahuan Desert of Sothern New México and Western Texas. The Southwestern Naturalist, 56 (1), 44-53. https://doi.org/10.1894/JC-31.1

Sauer, R. J. (1968) Two new species of crab spiders (Araneae:Thomisidae) from North Dakota. The Canadian Entomologist, 100 (11), 1148-1150. https://doi.org/10.4039/Ent1001148-11

Sauer, R. J., Platnick, N. I. (1972) The crab spiders genus Ebo (Araneidae:Thomisidae) in the United States \& Canada. The Canadian Entomologist, 104 (1), 35-60. https://doi.org/10.4039/Ent10435-1

Schick, R. X. (1965) The crab spiders of California (Araneae:Thomisidae). Bulletin of the American Museum Natural History, 129, 1-180.

Schmidt, R. H. Jr. (1979) A climatic delineation of the "real" Chihuahuan desert. Journal of Arid Environments, 2, 243-250. https://doi.org/10.1016/S0140-1963(18)31774-9

Schmidt, R. H. Jr., Marston, R. A. (1981) Los Médanos de Samalayuca, Chihuahua, México. New Mexico Journal of Science, 21 (2), 21-27.

Slowik, J. (2009) A review of the cellar spider genus Psilochorus Simon 1893 in America north of Mexico (Araneae:Pholcidae). Zootaxa, 2144, 1-53.

Soberón, J. M., Llorente, J. (1991) The use of species accumulation function for the prediction of species richness. Conservation Biology, 7 (3), 480-488.

Sørensen, L. L. (2003) Stratification of the spider fauna in a Tanzania forest. Pp: 92-101. In: Y. Basset, V. Novotny, S. E. Miller, R. L. Kitching (Eds.). Arthropods of tropical forest: spatio-temporal dynamics and resource use in the canopy. Cambridge University Press, United Kingdom.

Ubick, D., Cushing, P., Paquin, P., Roth, V. (2017) Spiders of North America: an identification manual. 2nd Edition. American Arachnological Society, Keene, New Hampshire, U.S.A.

Valdez-Mondragón, A., Cortez-Roldán, M. R., Juárez-Sánchez, A. R., Solís-Catalán, K. P. (2018) A new species of Loxosceles Heineken \& Lowe (Araneae, Sicariidae), with updated distribution records and biogeographical comments for the species from Mexico, including a new record of Loxosceles rufescens (Dufour). ZooKeys, 802, 39-66. https://doi.org/10.3897/zookeys.802.28445

Wheater, C. P., Cullen, W. R., Bell, J. R. (2000) Spider communities as tools in monitoring reclaimed limestone quarry landforms. Landscape Ecology, 15 (5), 401-406.

World Spider Catalog (2019) Natural History Museum Bern. Disponible en: http://www.wsc.nmbe.ch, versión 20.0 . 